\title{
IDAHO NUCLEAR TECHNOLOGY AND ENGINEERING CENTER LOW-ACTIVITY WASTE PROCESS TECHNOLOGY PROGRAM FY-98 STATUS REPORT
}

\author{
A. K. Herbst \\ J. A. McCray \\ A. Z. Rogers \\ R. F. Simmons \\ S. J. Palethrope
}


INEEL/EXT-99-00063

\title{
IDAHO NUCLEAR TECHNOLOGY AND ENGINEERING CENTER LOW-ACTIVITY WASTE PROCESS \\ TECHNOLOGY PROGRAM FY-98 STATUS REPORT
}

\author{
A. K. Herbst \\ J. A. McCray \\ A. Z. Rogers \\ Lockheed Martin Idaho Technologies Company \\ R. F. Simmons \\ S. J. Palethorpe \\ AEA Technologies
}

Published March 1999

Idaho National Engineering and Environmental Laboratory

Lockheed Martin Idaho Technologies Company

Idaho Falls, Idaho 83415

Prepared for the

U.S. Department of Energy

Under DOE Idaho Operations Office

Contract DE-AC07-94ID13223 


\begin{abstract}
The Low-Activity Waste Process Technology Program at the Idaho Nuclear Technology and Engineering Center (INTEC) anticipates that large volumes of low-level/low-activity wastes will need to be grouted prior to nearsurface disposal. During fiscal year 1998, three grout formulations were studied for low-activity wastes derived from INTEC liquid sodium-bearing waste. Compressive strength and leach results are presented for phosphate bonding cement, acidic grout, and alkaline grout formulations. In an additional study, grout formulations are recommended for stabilization of the INTEC underground storage tank residual heels.
\end{abstract}




\section{SUMMARY}

The general purpose of the Low-Activity Waste Process Technology Program is to solidify and stabilize the liquid low-activity wastes (LAW) generated at the Idaho Nuclear Technology and Engineering Center (formerly the Idaho Chemical Processing Plant). It is anticipated that LAW will be produced from the following: (1) chemical separation of the tank farm liquid sodiumbearing waste, (2) chemical separation of dissolved aluminum and zirconium calcines, and (3) facility decontamination and process equipment wastes. Grout formulation studies for sodium-bearing LAW continued this fiscal year. A second task was to select a grout formulation to solidify potential process residual heels in the tank farm vessels when the vessels are closed.

Three grout formulations were studied this year with simulated wastes. Phosphate bonding cement was used with acidic sodium-bearing LAW. The grout passed strength tests, but mixes were difficult to control flash set. Acidic sodium-bearing LAW was grouted at $\mathrm{pH}>1$ and passed initial compression tests, but failed the immersion test. The third formulation, designed by AEA Technology of the UK, utilizes LAW adjusted to a $\mathrm{pH}<12$. These alkaline grouts successfully passed all stability/integrity tests. The specifics of these formulation tests along with a nitrate leach test and dimensional stability test are presented.

For underground storage tank closure, various grout formulations were tested with various tanks heel concentrations. It was determined that the heel would need to be rinsed at least three times to reduce the acidity to prevent grout decomposition and that the Savannah River reducing grout could be used with this heel.

During FY-99, an alternative method for grouting sodium-bearing waste will be studied. In this process, only cesium would be separated resulting in a transuranic waste. A grout formulation or other solidification method will be studied to meet the Waste Isolation Pilot Plant acceptance criteria. Grouting methods will be studied for facility decontamination and process equipment wastes. The international contract with AEA Technologies will continue with these latter waste streams. Additionally, a lab scale mock-up of the recommended tank heel grouts will be prepared. 


\section{ACKNOWLEDGMENTS}

The authors express their gratitude to the following persons for their assistance in this program:

Dr. Barry Scheetz, Materials Research Laboratory, Pennsylvania State University

Darrell Dietz, Ash Grove Cement Company

Oliver Hartwig, Technical Editor, Lockheed Martin Idaho Technology Co.

Bill Holtzscheiter, Tanks Focus Area, Westinghouse Savannah River Co. 


\section{NOMENCLATURE}

Acid Grout

Alkaline Grout

Al LAW

Blast Furnace Slag

Calcination

Cement

Denitration

Fly Ash

Grout

Leaching

Low-Activity Waste

Na LAW

Portland Cement
A grout formulation where the acidity of the waste is kept to a $\mathrm{pH}$ less than 1.0 and mixed with a 3:1:1 blend of blast furnace slag, portland cement, and fly ash.

A grout formulation where the waste is rendered basic $(\mathrm{pH}>12)$ and mixed with a 9:1 blend of blast furnace slag and portland cement.

Low-activity waste derived from dissolved, separated aluminum calcine.

A finely ground non-metallic waste produce developed in the manufacture of pig iron, consisting basically of a mixture of lime, silica, and alumina, the same oxides that make up portland cement, but not in the same proportions or forms.

The process of converting a liquid to a solid product called calcine.

Refers to type I/II portland cement.

A thermal process to destroy the nitrate content of the waste.

A pozzolan of finely divided residue that results from the combustion of ground or powdered coal. Class $\mathrm{C}$ fly ash may contain $10 \%$ lime, has cementitious properties, and reacts with water to form a solid. Class $\mathrm{F}$ fly ash does not use water and aids in grout flow.

A mixture of portland cement, other powdered additives, waste, and water. It may contain fine-grained sand and does not include large aggregate material. For this study, grouting is the process of solidifying and stabilizing low-level waste in cement based materials.

The process whereby a liquid agent will dissolve hazardous materials within a waste mass and transport these materials through the mass and beyond. The most widely used laboratory leaching test is the TCLP (Toxic Characteristic Leaching Procedure) specified by the EPA in several regulations. For many treated and untreated wastes, the results of this test determine whether the EPA considers the material toxic or not.

Low-level waste derived from the solvent extraction, ion exchange, and chemical extraction separation processes on the tank farm sodiumbearing waste and on the dissolved calcines.

Low-activity waste derived from sodium-bearing waste.

The product obtained by pulverizing clinker consisting essentially of hydraulic calcium silicates. 


\section{Pozzolan}

\section{3-Way Blend}

Solidification

Stabilization

Water-to-Cement Ratio

Waste Form

Waste Loading

Vitrify

$\mathbf{V o l}_{\text {grout }} / \mathbf{V o l}_{\text {sim }}$
A siliceous or siliceous and aluminous material that reacts with liquid calcium hydroxide in the cement gel to form compounds possessing cementitious properties.

A 1:1:1 blend of portland cement, blast furnace slag, and coal fired power plant fly ash on a mass basis.

The process of producing from liquid, sludge, or loose solids a more or less monolithic structure having some integrity. Occasionally, solidification may refer to the process that results in a soil-like material rather than a monolithic structure. Solidification does not necessarily reduce leaching of hazardous materials. However, when a waste is solidified, its mass and structure are altered, decreasing migration of solutions within the mass.

Generally refers to a purposeful chemical reaction that has carried out to make waste constituents less leachable. This is accomplished by chemically immobilizing hazardous materials or reducing their solubility by a chemical reaction.

Defined as the mass of the water divided by the mass of the cements used (portland cement plus cement additives). In the case of the three way blends of portland cement, blast furnace slag, and fly ash, the mass of the water was divided by the total mass of the three cementing agents. The mass of the waste is not included in this calculation.

The final product for long-term storage. This includes the solidified/stabilized waste as well as the container. The waste form must pass extensive qualification testing prior to release for storage.

The mass weight percent of the waste in the total mass of the mixed grout.

The process of placing waste material in a glass form. This is a thermal process where the waste material is placed in a melter with glass beads or frit, then heated together, poured into a storage container, and cooled to a solid form.

The ratio of the volume of grout produced from a given volume of the original liquid low-level waste volume. For a ratio of 4 , the volume of grout would be 4 times greater than the original volume of the liquid waste. If the ratio is less than 1.0, the volume of grout would be less than the original liquid waste volume. An objective of the preconditioning and grouting processes is to optimize (reduce) this ratio; thus, reducing final repository costs based on volume.

Low-activity waste derived from dissolved, separated zirconium calcine. 


\section{CONTENTS}

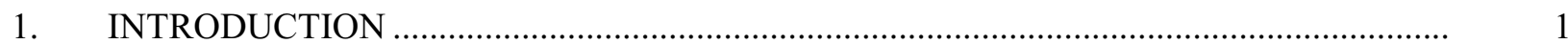

2. PHOSPHATE BONDING CEMENT COMPARISON STUDY FOR INTEC

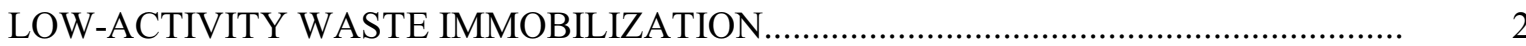



2.2 Portland Cement Based Grout Immobilization Process Description........................... 3

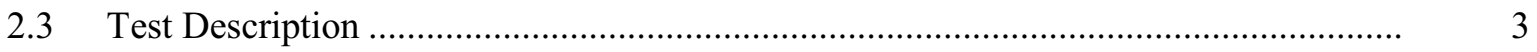

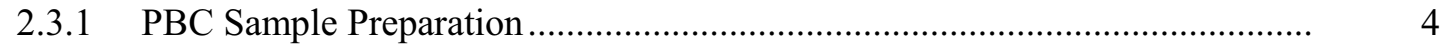

2.3.2 Portland Cement Based Grout Sample Preparation............................................ 8

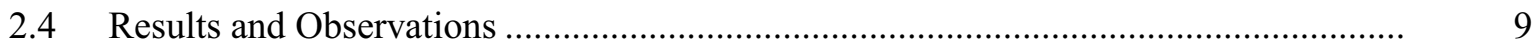

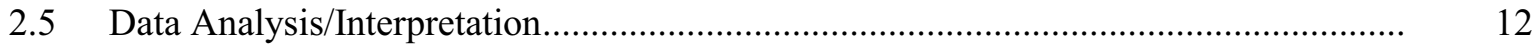

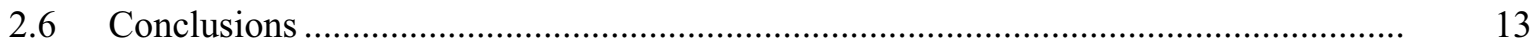

3. TANK FARM CLOSURE GROUTING DEVELOPMENT UPDATE.................................... 14





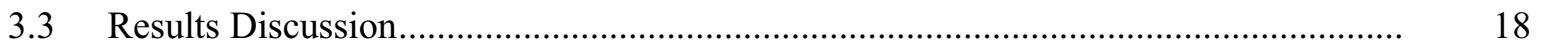

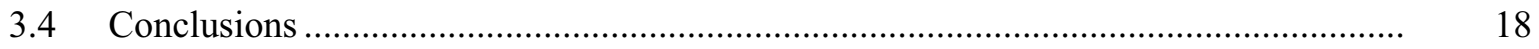

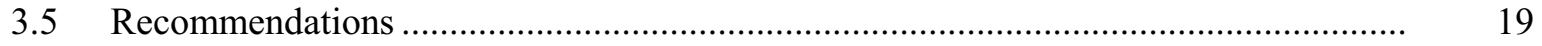

4. CEMENTITIOUS IMMOBILIZATION OF STRONGLY ACIDIC, NITRATE SALT









4.3.1 Portland Cement, Type I/II ..................................................................... 22

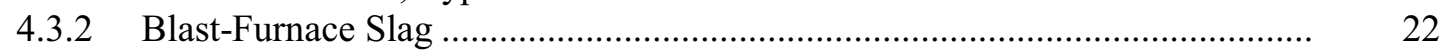

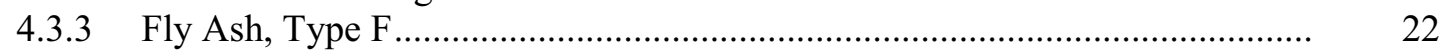

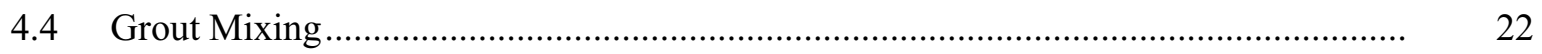




4.5.1 NRC Position on Grout Performance ............................................................. 23



4.5.3 Nitrate Leach Test ............................................................................ 24

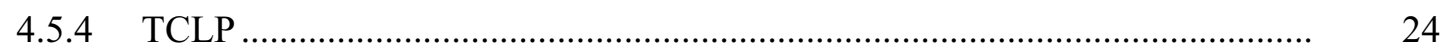

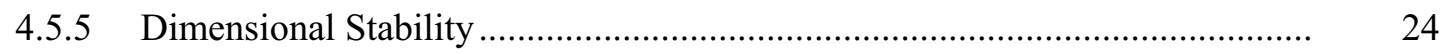

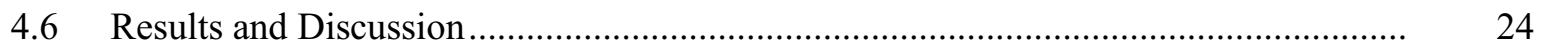



4.6.2 Nitrate Leach Test ............................................................................... 26



4.6.4 Dimensional Stability ....................................................................... 27



5. SOLIDIFICATION OF ACIDIC, HIGH-SODIUM LOW LEVEL WASTE AT THE IDAHO NATIONAL ENGINEERING AND ENVIRONMENTAL LABORATORY ...................... 29

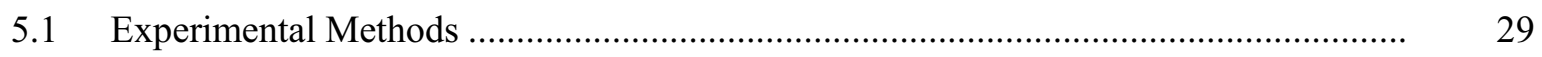

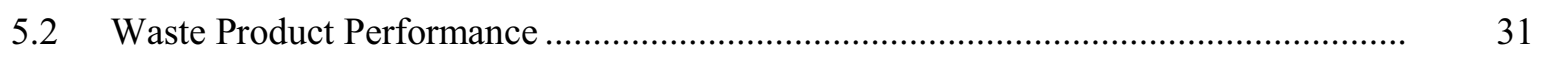

5.2.1 Small-scale studies at INEEL ..................................................................... 31

5.2.2 Large-scale studies at AEA Technology (Winfrith, UK) ............................... 32

5.3 Implications for Waste Processing ....................................................................

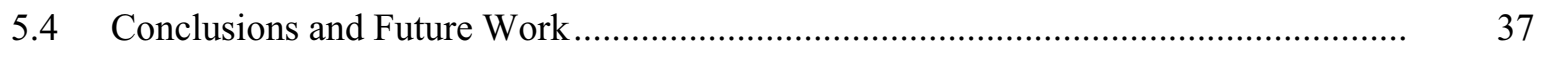

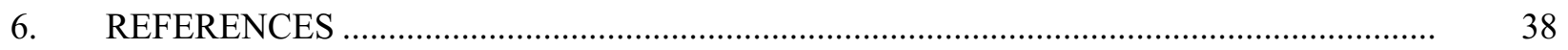

\section{FIGURES}

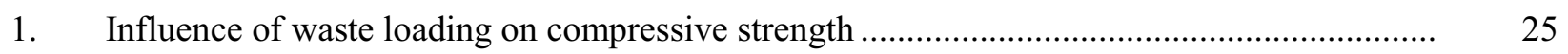

2. Cored full-scale $(200 \mathrm{~L})$ cemented sodium-bearing waste ............................................... 35

\section{TABLES}



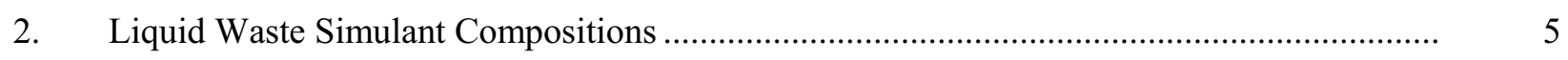

3. Denitrated Waste Simulant Solids Compositions …....................................................

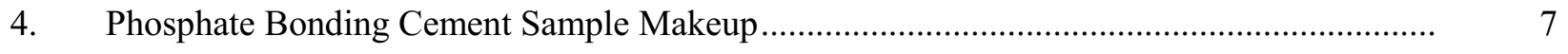

5. Portland Cement Based Grout Sample Makeup …..................................................... 8 
6. Test Results for Phosphate-Bonding Cement Samples................................................... 10



8. Test Grout Formulations and Makeups of Submersion Solutions …..................................... 15

9. Tank Farm Simulant Chemical Composition (Prior to Dilution) …...................................... 15

10. Grout Sample Compressive Strength and Corrosion Results …....................................... 17

11. Waste Simulant Compositions. Concentrations of major species in the highly acidic, alkali



12. Nitrate leachability index versus waste weight percent. The leach indexes for acidic grout are similar to values obtained for grouted waste forms using preconditioned waste solutions .....

13. TCLP Results. Grouted waste forms pass TCLP at three varied waste loadings .................... 27

14. Percent length change over 28-day period. Acidic grout had the greatest amount of length

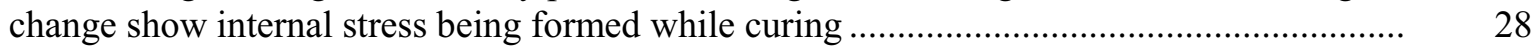



16. Estimated grout formulations to produce a cubic meter of grouted low-activity waste .......... 31

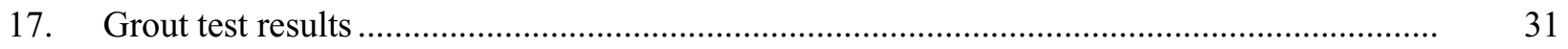

18. Test results for the full-scale (200-litre) cementation trial on alkaline sodium bearing waste simulant grout formulation

19. Pore size distribution results for full-scale (200-litre) cementation trial on alkaline sodium bearing



20. Testing cores from full-scale (200-litre) cementation trial on alkaline sodium bearing waste simulant grout formulation 


\section{IDAHO NUCLEAR TECHNOLOGY AND ENGINEERING CENTER LOW-LEVEL WASTE PROCESS TECHNOLOGY PROGRAM FY-98 STATUS REPORT}

\section{INTRODUCTION}

The High-Level Waste Program Plan for the Idaho Nuclear Technology and Engineering Center (INTEC) calls for the sodium-bearing liquid waste in the tank farm to be separated into high-activity and low-activity radioactive wastes. ${ }^{1}$ The calcine in the storage bins will be retrieved, dissolved in nitric acid, and separated. The small volume, high-activity waste will be vitrified and disposed of in a geological repository. The large volume, low-activity waste (LAW) will be grouted and either returned to the tank farm vessels or disposed of in drums. In addition to the LAW derived from separations, facility decontamination and the process equipment waste system will continue to generate low-level waste (LLW) liquids to be grouted. These decontamination and process equipment solutions are also a form of sodium-bearing waste. This research, performed by the LAW Process Technology group, provides data on the various LAW/LLW alternatives being proposed by the Department of Energy (DOE) High-Level Waste and Facilities Disposition Environmental Impact Statement.

The fiscal year 1998 work is discussed in four main sections. The first section presents comparison tests performed with phosphate bonding cement and portland cement for immobilizing three different types of simulated INTEC low-activity waste (LAW). The next section covers the tests to determine the optimum grout formulation for underground storage tank heel closure and in particular the performance of Savannah River's reducing grout formulation with projected INTEC tank heels. Section three reports on the performance of grouts prepared by directly adding liquid acidic waste $(\mathrm{pH}<1.0)$ to the cement powders. The fourth section presents the work completed by AEA Technologies in the United Kingdom to compare acidic grouts with their alkaline grout formulation $(\mathrm{pH}>12)$ where the acidic waste is made basic and added to the cement powders.

The criteria for an acceptable grout formulation and wasteform are governed by the Department of Energy and augmented with guidelines from the U.S. Nuclear Regulatory Commission. These documents are Department of Energy Order 5820.2a, "Radioactive Waste Management," and the "Technical Position on Waste Form," published by the Low-Level Waste Management Branch of the U.S. Nuclear Regulatory Commission (NRC). ${ }^{2}$ The NRC guidelines are specifically directed at grouted wasteforms and contain definitive qualifications in the areas of compressive strength, leach resistance, thermal cycling, immersion testing, and irradiation. 


\section{PHOSPHATE BONDING CEMENT COMPARISON STUDY FOR INTEC LOW-ACTIVITY WASTE IMMOBILIZATION}

Radioactive wastes at the INTEC consist of approximately $6800 \mathrm{~m}^{3}$ aqueous and $3800 \mathrm{~m}^{3}$ of dry calcine solid. Current plans call for the liquid waste to be either directly calcined, or separated into highactivity and low-level streams using solvent extraction and ion exchange technologies. The solid calcine is to be dissolved in nitric acid and similarly separated. The smaller volume high-activity waste stream will be vitrified and disposed of in a geological repository. The larger volume LAW stream will be grouted and sent to a near-surface low-level disposal facility. ${ }^{3}$

The projected LAW will consist of three distinct streams: Na LAW, Al LAW, and Zr LAW. Na LAW will be from the separation treatment of the existing liquid waste (assuming that is the selected treatment method), which is high in sodium content. Al LAW and Zr LAW will be from the dissolution and separation treatment of aluminum and zirconium calcine types, respectively. Each calcine type has a relatively high concentration of the respective metal ion. All three LAW waste types will be acidic, high in nitrate concentration, and contain toxic metals and/or radionuclides. It is uncertain at this point in time whether the liquid LAW will be grouted directly, or thermally solidified and denitrated, and then grouted.

Currently, a research program is in place to develop grout formulations and processing methods for the eventual immobilization treatment of these low-level wastes. The emphasis of this program has been on grout formulations using ordinary portland cement (OPC), blast furnace slag (BFS), and fly ash.

Phosphate bonding cement (PBC) is being developed at Argonne National Laboratory (ANL) as an alternative method for the immobilization and stabilization of Department of Energy (DOE) LAW streams. This technology involves the reaction of magnesium oxide $(\mathrm{MgO})$ with monopotassium phosphate $\left(\mathrm{KH}_{2} \mathrm{PO}_{4}\right)$ and water to form a dense monolithic solid. Fly ash can also be added to increase the mechanical strength of the magnesium potassium phosphate binder. In addition to being microencapsulated in the physical matrix of the solid waste form, hazardous and radioactive components are reported to react with phosphate ions to form relatively insoluble products. ${ }^{4}$

This testing investigates the potential applicability of PBC technology for immobilizing the three different types of future INTEC LAW. For each waste type, immobilization testing was performed for both untreated (acidic liquid with high nitrate content) and treated (denitrated solid) forms. Similar samples of the same waste loadings (based on weight percent) were also prepared, where applicable, using the current optimum portland cement based grout formulation for each waste type. For these initial scoping tests, the two immobilization technologies are compared directly for INTEC LAW treatment, based only on sample compression strengths and Toxicity Characteristics Leach Procedures (TCLP) results. The minimum acceptable wasteform compression strength is $500 \mathrm{psig}$. The Universal Treatment Standards TCLP limits for hazardous metals of concern are presented in Table 1 ( 40 CFR 268.48).

\subsection{PBC Immobilization Process Description}

The formulation and procedure for preparing PBC samples was obtained during an information gathering visit to Argonne National Laboratory. The PBC process experts there provided the required instructions for general wasteform preparation, and demonstrated the preparation of several samples using sodium nitrate and nitric acid as waste simulants. 
Table 1. Universal Treatment Standards TCLP Limits.

\begin{tabular}{|c|c|}
\hline Species & Nonwastewater Standard (mg/l) \\
\hline $\mathrm{As}$ & 5.0 \\
\hline $\mathrm{Ba}$ & 7.6 \\
\hline $\mathrm{Cd}$ & 0.19 \\
\hline $\mathrm{Cr}$ & 0.86 \\
\hline $\mathrm{Pb}$ & 0.37 \\
\hline $\mathrm{Hg}$ & 0.20 \\
\hline $\mathrm{Ni}$ & 5.0 \\
\hline $\mathrm{Se}$ & 0.16 \\
\hline $\mathrm{Ag}$ & 0.30 \\
\hline
\end{tabular}

PBC wasteform binding material is generally composed of equal moles of calcined magnesium oxide $\left(\mathrm{MgO}\right.$, prepared by Argonne) and monopotassium phosphate $\left(\mathrm{KH}_{2} \mathrm{PO}_{4}\right)$. PBC dry powder is made by blending equal amounts (by weight) of binding material and fly ash. Sufficient water is added to the dry powder to approximately satisfy the chemical reaction equation:

$$
\mathrm{MgO}+\mathrm{KH}_{2} \mathrm{PO}_{4}+5 \mathrm{H}_{2} \mathrm{O}=\mathrm{MgKPO}_{4} \cdot 6 \mathrm{H}_{2} \mathrm{O}
$$

Contaminant cations present will generally combine with abundant phosphate anions, forming relatively insoluble molecules. These contaminant molecules are also encapsulated in the magnesium phosphate matrix.

\subsection{Portland Cement Based Grout Immobilization Process Description}

Current development work for LAW immobilization at the INTEC is primarily concentrated on the use of portland cement based grouts, consisting of different mass ratio blends of OPC, fly ash, and BFS. Water addition to these powder blends causes hydration and pozzolanic reactions, eventually resulting in the formation of a solid monolithic wasteform. Both fly ash and BFS decrease permeability, increases mix fluidity, and lower initial heat evolution. BFS also aids in the retention of reducible mobile species.

Contaminant cations present will generally combine with abundant hydroxide anions, forming relatively insoluble molecules. These contaminant molecules are also encapsulated in the cement matrix.

\subsection{Test Description}

For both PBC and portland cement based grout immobilization technologies, 2-inch cube samples were prepared to simulate the immobilization of three different types of INTEC mixed waste (LAW fractions resulting from radionuclide separation operations performed on sodium-bearing liquid waste, dissolved aluminum calcine, and dissolved zirconium calcine). For each waste type, samples were prepared for both immobilization technologies, for both untreated (high nitrate acidic solutions) and 
treated (denitrated solid) waste simulant. The anticipated average LAW compositions (excluding radioactive components), and the composition of the liquid simulants used in this study, are presented in Table 2. For the Na LAW simulant only, the solution used was double the concentration of the actual waste in all respective non-radioactive ions. Waste loadings were calculated by doubling the weight fraction of the double-strength simulant contained in the respective samples. Due to the differences in density of the Na LAW simulant and water, these stated waste loadings are slightly higher than actual.

Analytical results for the denitrated solid forms of the three LAW simulants are presented in Table 3. These solid wastes were generated by thermally calcining (at $650^{\circ} \mathrm{C}$ for 2 hours) the same liquid waste simulants listed in Table 2. At this elevated temperature, the solutions are dehydrated and the contained nitrates should be volatilized or decomposed. Note, however, that the "denitrated" Na LAW solids remained extremely high in nitrate concentration.

\subsubsection{PBC Sample Preparation}

The makeup recipes for the PBC samples are presented in Table 4. These samples were each allowed to cure for a period of at least 20 days. Following the curing period, each sample was subjected to compression strength testing and to a TCLP leach test.

Individual $\mathrm{PBC}$ samples were generally prepared by blending the solids (denitrated solid waste simulant and/or $\mathrm{MgO}, \mathrm{KH}_{2} \mathrm{PO}_{4}$, and fly ash) together in one plastic dish, and the liquids (liquid waste simulant and/or water) together in another. The liquid was then added to the solids, and the slurry was continuously and rapidly mixed. As the $\mathrm{MgO}$ eventually dissolves in the solution, allowing subsequent reaction with the phosphate ions, the slurry begins to thicken and heat is evolved due to the exothermic reactions taking place. The mixture was poured into a plastic mold after it had thickened considerably, but was still fluid enough to pour. The mold was completely filled and excess grout was scraped off, leaving the remaining grout flush with the mold opening surface. The sample was then placed in a styrofoam insulated container for several hours to minimize reaction heat loss.

Deviations from this general procedure and/or makeup recipe were required for the generation of several samples. Samples 1A through 4A were initially prepared to evaluate the potential for higher liquid addition and/or increased liquid waste fraction. As sample generation progressed, it became obvious that the chemical reactions associated with the matrix formation in this process were highly sensitive to the $\mathrm{pH}$ of the waste. This is evident in the decrease of stir time required prior to initial set as a function of increased acidity of the mix (Table 4, samples 6A, 7A, and 8A). The reduction in required stir time is assumed to be due to the respective increased rates of $\mathrm{MgO}$ dissolution. As a result of samples $9 \mathrm{~A}$ and $10 \mathrm{~A}$ requiring excessive periods of stir time, sample $11 \mathrm{~A}$ was prepared using phosphoric acid in replacement of the $\mathrm{KH}_{2} \mathrm{PO}_{4}$. This dramatic increase in the mixture acidity, however, caused that sample to set almost immediately upon the addition of the liquid solution to the blended solids. In fact, sample $11 \mathrm{~A}$ had solidified before it could be placed into a mold, and no qualification testing was performed on it. The remainder of the samples prepared with solid waste simulant used different ratios of phosphoric acid and $\mathrm{KH}_{2} \mathrm{PO}_{4}$, in an attempt to attain a reasonable required stir time ( $<15$ minutes). These ratios were prepared by separately reacting phosphoric acid with different amounts of potassium carbonate $\left(\mathrm{K}_{2} \mathrm{CO}_{3}\right)$, mixing the reaction product $\left(\mathrm{KH}_{2} \mathrm{PO}_{4} /\right.$ phosphoric acid mixture) with the other liquids, and finally mixing the solution with the blended solids. 
Table 2. Liquid Waste Simulant Compositions.

\begin{tabular}{|c|c|c|c|c|c|c|}
\hline \multirow[b]{3}{*}{ Species } & \multicolumn{6}{|c|}{ Composition (M) } \\
\hline & \multicolumn{2}{|c|}{ Na LAW } & \multicolumn{2}{|c|}{ Al LAW } & \multicolumn{2}{|c|}{ Zr LAW } \\
\hline & Actual & Simulant $^{*}$ & Actual & Simulant & Actual & Simulant \\
\hline $\mathrm{H}$ & $7.70 \mathrm{E}-01$ & & $1.57 \mathrm{E}+00$ & & $1.61 \mathrm{E}+00$ & \\
\hline $\mathrm{Al}$ & $3.37 \mathrm{E}-01$ & $6.85 \mathrm{E}-01$ & $3.86 \mathrm{E}-01$ & $3.81 \mathrm{E}-01$ & $1.80 \mathrm{E}-01$ & $1.85 \mathrm{E}-01$ \\
\hline As & $3.11 \mathrm{E}-05$ & $4.81 \mathrm{E}-05$ & 0 & $\mathrm{Nd}^{* *}$ & 0 & nd \\
\hline $\mathrm{Ba}$ & $3.21 \mathrm{E}-05$ & $5.90 \mathrm{E}-05$ & 0 & nd & 0 & $2.40 \mathrm{E}-05$ \\
\hline $\mathrm{B}$ & $9.47 \mathrm{E}-03$ & $2.12 \mathrm{E}-02$ & $3.74 \mathrm{E}-03$ & $3.69 \mathrm{E}-03$ & $3.50 \mathrm{E}-02$ & $3.26 \mathrm{E}-02$ \\
\hline $\mathrm{Cd}$ & $1.47 \mathrm{E}-03$ & $2.73 \mathrm{E}-03$ & 0 & $7.92 \mathrm{E}-07$ & $3.45 \mathrm{E}-03$ & $3.27 \mathrm{E}-03$ \\
\hline $\mathrm{Ca}$ & $2.79 \mathrm{E}-02$ & $3.97 \mathrm{E}-02$ & 0 & $1.69 \mathrm{E}-03$ & $3.66 \mathrm{E}-01$ & $2.74 \mathrm{E}-01$ \\
\hline $\mathrm{Cl}$ & $1.53 \mathrm{E}-02$ & na*** & 0 & na & 0 & na \\
\hline $\mathrm{Cr}$ & $1.95 \mathrm{E}-03$ & $3.60 \mathrm{E}-03$ & 0 & $1.54 \mathrm{E}-05$ & 0 & $2.69 \mathrm{E}-06$ \\
\hline $\mathrm{Cs}$ & $5.79 \mathrm{E}-10$ & $\mathrm{Na}$ & $7.05 \mathrm{E}-10$ & na & $1.21 \mathrm{E}-09$ & na \\
\hline $\mathrm{F}$ & $3.63 \mathrm{E}-02$ & $\mathrm{Na}$ & 0 & na & $5.66 \mathrm{E}-01$ & na \\
\hline $\mathrm{Fe}$ & $1.16 \mathrm{E}-02$ & $1.86 \mathrm{E}-02$ & $1.63 \mathrm{E}-03$ & $1.61 \mathrm{E}-03$ & $1.83 \mathrm{E}-03$ & $3.32 \mathrm{E}-03$ \\
\hline $\mathrm{Pb}$ & $7.37 \mathrm{E}-04$ & $1.40 \mathrm{E}-03$ & 0 & nd & 0 & nd \\
\hline $\mathrm{Mn}$ & $8.42 \mathrm{E}-03$ & $1.65 \mathrm{E}-02$ & 0 & $2.19 \mathrm{E}-06$ & $1.22 \mathrm{E}-04$ & $1.18 \mathrm{E}-04$ \\
\hline $\mathrm{Hg}$ & $5.73 \mathrm{E}-04$ & $8.28 \mathrm{E}-04$ & 0 & 8.47E-09 & 0 & nd \\
\hline Mo & $3.68 \mathrm{E}-04$ & $7.10 \mathrm{E}-04$ & 0 & nd & 0 & nd \\
\hline $\mathrm{Ni}$ & $8.42 \mathrm{E}-04$ & $1.68 \mathrm{E}-03$ & 0 & $1.24 \mathrm{E}-05$ & 0 & nd \\
\hline $\mathrm{NO}_{3}$ & $2.67 \mathrm{E}+00$ & $\mathrm{Na}$ & $2.75 \mathrm{E}+00$ & na & $2.75 \mathrm{E}+00$ & na \\
\hline $\mathrm{PO}_{4}$ & 0 & $\mathrm{Na}$ & 0 & na & $5.65 \mathrm{E}-04$ & na \\
\hline $\mathrm{K}$ & $1.08 \mathrm{E}-01$ & $2.79 \mathrm{E}-01$ & 0 & $7.19 \mathrm{E}-04$ & $6.49 \mathrm{E}-03$ & $1.29 \mathrm{E}-02$ \\
\hline $\mathrm{Se}$ & $1.50 \mathrm{E}-05$ & $2.91 \mathrm{E}-05$ & 0 & nd & 0 & nd \\
\hline $\mathrm{Ag}$ & $1.21 \mathrm{E}-05$ & $6.02 \mathrm{E}-06$ & 0 & nd & 0 & $2.29 \mathrm{E}-04$ \\
\hline $\mathrm{Na}$ & $1.10 \mathrm{E}+00$ & $1.71 \mathrm{E}+00$ & $9.03 \mathrm{E}-02$ & $9.17 \mathrm{E}-02$ & $1.18 \mathrm{E}-01$ & $1.20 \mathrm{E}-01$ \\
\hline $\mathrm{SO}_{4}$ & $2.63 \mathrm{E}-02$ & na & $2.72 \mathrm{E}-03$ & na & $1.65 \mathrm{E}-03$ & na \\
\hline $\mathrm{Zr}$ & $5.26 \mathrm{E}-04$ & na & 0 & na & $8.50 \mathrm{E}-02$ & na \\
\hline $\begin{array}{ll}* & \text { The Na LAW s } \\
& \text { a LAW). } \\
* * & \text { nd-not detected } \\
* * * & \text { na- sample not }\end{array}$ & $\begin{array}{l}\text { r this testing } \\
\text { at element }\end{array}$ & ade double st & (twice the co & ation of the $\mathrm{r}$ & ive ions in th & \\
\hline
\end{tabular}


Table 3. Denitrated Waste Simulant Solids Compositions.

\begin{tabular}{|c|c|c|c|}
\hline \multirow{2}{*}{ Species(Majors) } & \multicolumn{3}{|c|}{ Composition (Wt \%) } \\
\cline { 2 - 4 } & Na LAW & Al LAW & Zr LAW \\
\hline $\mathrm{Al}$ & 7.2 & 36.0 & 10.2 \\
\hline $\mathrm{B}$ & 0.09 & 0.12 & 0.58 \\
\hline $\mathrm{Cd}$ & 0.36 & - & 2.2 \\
\hline $\mathrm{Ca}$ & 0.7 & 0.28 & 21.0 \\
\hline $\mathrm{Cr}$ & 0.07 & - & - \\
\hline $\mathrm{Fe}$ & 0.48 & 0.5 & 0.2 \\
\hline $\mathrm{Pb}$ & 0.12 & - & - \\
\hline $\mathrm{Mg}$ & 0.07 & - & - \\
\hline $\mathrm{NO}$ & 47.0 & 0.6 & 1.3 \\
\hline $\mathrm{K}$ & 1.7 & 0.1 & 0.7 \\
\hline $\mathrm{Na}$ & 18.3 & 7.0 & 5.8 \\
\hline $\mathrm{Zr}$ & - & - & 18.0 \\
\hline
\end{tabular}


Table 4. Phosphate Bonding Cement Sample Makeup

\begin{tabular}{|c|c|c|c|c|c|c|c|c|c|}
\hline \multirow[b]{2}{*}{$\begin{array}{c}\text { Sample } \\
\text { Num. }\end{array}$} & \multirow[b]{2}{*}{$\begin{array}{l}\text { Waste } \\
\text { Type } \\
\end{array}$} & \multicolumn{7}{|c|}{ Sample Makeup Ingredients (g) } & \multirow{2}{*}{$\begin{array}{c}\text { Stir } \\
\text { Time } \\
\text { (min.) }\end{array}$} \\
\hline & & MgO & $\mathrm{KH}_{2} \mathrm{PO}_{4}$ & $\mathrm{H}_{3} \mathbf{P O}_{4}$ & $\mathrm{~K}_{2} \mathrm{CO}_{3}$ & $\begin{array}{l}\text { Fly } \\
\text { Ash }\end{array}$ & $\begin{array}{c}\text { Waste } \\
\text { Simulant } \\
\end{array}$ & $\mathrm{H}_{2} \mathbf{O}$ & \\
\hline \multicolumn{10}{|c|}{ Na LAW Simulant Samples } \\
\hline $1 \mathrm{~A}$ & Liquid ${ }^{*}$ & 14.5 & 49.2 & - & - & 63.8 & 127.5 & - & 3 \\
\hline $2 \mathrm{~A}$ & Liquid* & 14.5 & 49.2 & - & - & 63.8 & 76.5 & 51.0 & 16 \\
\hline $3 \mathrm{~A}$ & Liquid* & 21.0 & 70.1 & - & - & 91.1 & 78.1 & - & 0.5 \\
\hline $4 \mathrm{~A}$ & Liquid* & 18.0 & 60.0 & - & - & 78.0 & 104.2 & - & 6.5 \\
\hline $5 \mathrm{~A}$ & Liquid* & $29^{* *}$ & 49.2 & - & - & 49.3 & 127.6 & - & 1.5 \\
\hline $6 \mathrm{~A}$ & Liquid $^{*}$ & 21.5 & 71.8 & - & - & 93.5 & 28.0 & 65.3 & 45 \\
\hline $7 \mathrm{~A}$ & Liquid* & 21.5 & 71.8 & - & - & 93.3 & 42.0 & 51.3 & 35 \\
\hline $8 \mathrm{~A}$ & Liquid* & 21.5 & 71.8 & - & - & 93.3 & 56.0 & 37.3 & 26 \\
\hline $9 \mathrm{~A}$ & Solid & 18.4 & 61.7 & - & - & 80.1 & 39.8 & 80.1 & 90 \\
\hline $10 \mathrm{~A}$ & Solid & 16.1 & 53.7 & - & - & 69.8 & 70.6 & 69.8 & 120 \\
\hline $11 \mathrm{~A}$ & Solid & 16.1 & - & 38.7 & - & 69.8 & 70.6 & 65.7 & $<1$ \\
\hline $12 \mathrm{~A}$ & Solid & 18.4 & - & 50.8 & 15.7 & 80.1 & 38.7 & 75.6 & 14 \\
\hline $13 \mathrm{~A}$ & Solid & 15.8 & - & 43.6 & 13.5 & 68.7 & 67.5 & 64.7 & 65 \\
\hline \multicolumn{10}{|c|}{ Al LAW Simulant Samples } \\
\hline $14 \mathrm{~A}$ & Liquid & 20.7 & 69.3 & - & - & 90.0 & 54.0 & 36.0 & 28 \\
\hline $15 \mathrm{~A}$ & Liquid & 20.7 & 69.3 & - & - & 90.0 & 81.0 & 9.0 & 6.5 \\
\hline $16 \mathrm{~A}$ & Solid & 18.4 & - & 50.8 & 15.7 & 80.1 & 39.2 & 75.6 & 10 \\
\hline $17 \mathrm{~A}$ & Solid & 15.8 & - & 43.5 & 10.4 & 68.7 & 65.2 & 64.8 & 2 \\
\hline \multicolumn{10}{|c|}{ Zr LAW Simulant Samples } \\
\hline $18 \mathrm{~A}$ & Liquid & 20.7 & 69.3 & - & - & 90.0 & 54.0 & 36.0 & 27 \\
\hline $19 \mathrm{~A}$ & Liquid & 20.7 & 69.3 & - & - & 90.0 & 81.0 & 9.0 & 4 \\
\hline $20 \mathrm{~A}$ & Liquid & 31.1 & 69.3 & - & - & 79.6 & 81.0 & 9.0 & 4 \\
\hline $21 \mathrm{~A}$ & Solid & 18.4 & - & 50.8 & 15.7 & 80.1 & 39.2 & 75.6 & 2 \\
\hline $22 \mathrm{~A}$ & Solid & 15.8 & - & 43.5 & 18.0 & 68.7 & 65.2 & 64.8 & 3 \\
\hline $23 \mathrm{~A}$ & Solid & 18.4 & - & 50.8 & 20.9 & 80.1 & 38.6 & 75.6 & 6 \\
\hline
\end{tabular}


Poor initial PBC sample compression strength test results were obtained and communicated to the ANL process experts. The poor compression strengths were believed to be due to the $\mathrm{MgO}$ reacting with the free hydrogen ions (acid) in the waste, preventing the required reaction with the available phosphate ions. Samples 5A and 20A were prepared using double and 1.5 times the normal amounts of $\mathrm{MgO}$, respectively, to determine the impacts on compression strengths. In both cases, the excess $\mathrm{MgO}$ was premixed with the respective liquid to partially neutralize the acidic solution prior to adding the remaining blended powders. Additionally, in both sample cases, the amount of fly ash added was decreased by the same mass amount as the $\mathrm{MgO}$ had been increased, to maintain the desired waste loading.

\subsubsection{Portland Cement Based Grout Sample Preparation}

The makeup recipes used for the portland cement based grout samples are presented in Table 5 . These samples were allowed to cure for a period of at least 28 days. Following the curing period, each sample was subjected to compression strength testing and to a TCLP leach test.

Table 5. Portland Cement Based Grout Sample Makeup.

\begin{tabular}{|c|c|c|c|c|c|c|c|c|}
\hline \multirow[b]{2}{*}{$\begin{array}{l}\text { Sample } \\
\text { Num. }\end{array}$} & \multirow[b]{2}{*}{$\begin{array}{l}\text { Waste } \\
\text { Type }\end{array}$} & \multicolumn{6}{|c|}{ Sample Makeup Ingredients (g) } & \multirow[b]{2}{*}{ Solution $\mathrm{pH}$} \\
\hline & & $\mathrm{OPC}$ & BFS & Fly Ash & $\begin{array}{c}50 \% \\
\mathrm{NaOH} \\
\end{array}$ & $\begin{array}{c}\text { Waste } \\
\text { Simulant }\end{array}$ & $\mathrm{H}_{2} \mathrm{O}$ & \\
\hline \multicolumn{9}{|c|}{ Na LAW Simulant Samples } \\
\hline $1 \mathrm{~B}$ & Liquid* & 38.4 & 115.1 & 38.4 & 2.6 & 30.8 & 52.5 & 2.0 \\
\hline $2 \mathrm{~B}$ & Liquid* & 37.9 & 113.7 & 37.9 & 3.9 & 45.6 & 36.1 & 2.3 \\
\hline 3B & Liquid* & 36.6 & 109.9 & 36.6 & 5.2 & 60.6 & 26.0 & 1.7 \\
\hline $4 \mathrm{~B}$ & Liquid* & 36.6 & 109.9 & 36.6 & 7.5 & 86.6 & 0 & 1.3 \\
\hline $5 \mathrm{~B}$ & Solid & 47.0 & 47.0 & 47.0 & - & 35.3 & 72.0 & - \\
\hline $6 \mathrm{~B}$ & Solid & 40.0 & 40.0 & 40.0 & - & 64.6 & 72.0 & - \\
\hline \multicolumn{9}{|c|}{ Al LAW Simulant Samples } \\
\hline 7B & Liquid & 37.5 & 112.4 & 37.5 & 5.7 & 54.0 & 22.9 & 1.4 \\
\hline $8 \mathrm{~B}$ & Liquid & 36.5 & 109.7 & 36.5 & 8.3 & 80.2 & 0 & 1.7 \\
\hline $9 \mathrm{~B}$ & Solid & 47.0 & 47.0 & 47.0 & - & 35.0 & 74.0 & - \\
\hline $10 \mathrm{~B}$ & Solid & 39.0 & 39.0 & 39.0 & - & 61.6 & 75.0 & - \\
\hline \multicolumn{9}{|c|}{ Zr LAW Simulant Samples } \\
\hline $11 \mathrm{~B}$ & Liquid & 37.5 & 112.4 & 37.5 & 6.5 & 54.0 & 22.1 & 1.2 \\
\hline $12 \mathrm{~B}$ & Liquid & 36.5 & 109.7 & 36.5 & 9.6 & 80.2 & 0 & 1.5 \\
\hline $13 \mathrm{~B}$ & Solid & 47.0 & 47.0 & 47.0 & - & 35.0 & 74.0 & - \\
\hline $14 \mathrm{~B}$ & Solid & 39.0 & 39.0 & 39.0 & - & 61.6 & 75.0 & - \\
\hline
\end{tabular}


Portland cement based grout samples made using liquid LAW simulants were prepared using an optimized flowsheet and procedure. These samples were prepared using a 1:1:3 OPC to class F fly ash to BFS mass ratio. Liquid used for each sample (water and/or waste simulant) was $30 \mathrm{wt} \%$ of the total sample mass. The respective water used in any sample was initially mixed with the waste simulant. This solution was then partially neutralized with $50 \mathrm{wt} \% \mathrm{NaOH}$ solution to achieve a pH between 1 and 2 . The fly ash and BFS were then added to the solution, and the slurry stirred for approximately 2 minutes, to achieve further solution neutralization. The OPC was added last, stirred to achieve a homogenous mixture, and then immediately poured into the plastic mold. The mold was completely filled and excess grout was scraped off, leaving the remaining grout flush with the mold opening surface.

Portland cement based grout samples made with denitrated solid LAW simulants had been prepared and tested during a previous separate study using a standard flowsheet and procedure. ${ }^{5}$ These samples were prepared using a 1:1:1 OPC to fly ash to BFS mass ratio.

\subsection{Results and Observations}

Compression strength and TCLP test results for the PBC samples are presented in Table 6. Those results for the portland cement based grout samples are presented in Table 7 . Other test results and observations include:

1. The decrease in PBC sample weights, from generation until the end of the 20-day curing period, was less than $2 \%$ for all samples except numbers $1 \mathrm{~A}$ and $2 \mathrm{~A}$, which decreased in weight by $7.7 \%$ and $7.5 \%$, respectively. These same two were prepared using a $50 \%$ liquid mass fraction, considerably higher than that used in the preparation of the other PBC samples. (Sample $5 \mathrm{H}$ was prepared as duplicate of sample $1 \mathrm{~A}$, except with a higher $\mathrm{MgO}$ concentration.)

2. PBC binder reactions are extremely sensitive to the acidity or alkalinity of the added waste. This was indicated in the significant differences in stir time required before initial set with respect to waste simulant acidity. PBC flowsheet (or procedure) changes may be required with changing acidity or alkalinity of the waste being immobilized, to assure the required binder reactions take place in a reasonable time period.

3. Salt crystals "grew" from the single area surface exposed to the atmosphere on all PBC samples prepared using liquid waste simulant. The mass of salt crystal for each sample increased with increased waste loading and/or increased total liquid content (samples 1A and 2A had considerably more salt crystals form than any other). A single sample was taken of the combined crystals from these two samples, and analyzed. Results indicated that the crystals were composed mainly of potassium nitrate $(>90 \%)$. 
Table 6. Test Results for Phosphate-Bonding Cement Samples.

\begin{tabular}{|c|c|c|c|c|c|c|c|c|c|c|c|c|}
\hline \multirow[b]{2}{*}{$\begin{array}{c}\text { Sample } \\
\text { Num. }\end{array}$} & \multirow[b]{2}{*}{$\begin{array}{l}\text { Waste } \\
\text { Type }\end{array}$} & \multirow{2}{*}{$\begin{array}{l}\text { Waste } \\
\text { Load. } \\
\text { (wt\%) }\end{array}$} & \multirow{2}{*}{$\begin{array}{l}\text { Comp. } \\
\text { Strength } \\
\text { (psig) }\end{array}$} & \multicolumn{9}{|c|}{ TCLP Results (mg/L) } \\
\hline & & & & Ag & As & $\mathbf{B a}$ & Cd & $\mathbf{C r}$ & $\mathrm{Hg}$ & $\mathbf{N i}$ & $\mathbf{P b}$ & Se \\
\hline \multicolumn{13}{|c|}{ Na LAW Simulant Samples } \\
\hline $1 \mathrm{~A}$ & Liquid $^{*}$ & $100^{* *}$ & 180 & $\mathrm{nd}^{* * *}$ & nd & nd & nd & nd & 0.036 & 0.15 & nd & nd \\
\hline $2 \mathrm{~A}$ & Liquid $^{*}$ & $60^{* *}$ & 150 & nd & nd & nd & nd & nd & 0.066 & nd & nd & nd \\
\hline $3 \mathrm{~A}$ & Liquid $^{*}$ & $60^{* *}$ & 2220 & nd & nd & $\mathrm{U}$ & nd & nd & 0.089 & nd & nd & nd \\
\hline $4 \mathrm{~A}$ & Liquid $^{*}$ & $80^{* *}$ & 710 & nd & nd & nd & nd & nd & 0.047 & nd & nd & nd \\
\hline $5 \mathrm{~A}$ & Liquid $^{*}$ & $100^{* *}$ & 510 & nd & nd & nd & nd & nd & 0.022 & nd & nd & nd \\
\hline $6 \mathrm{~A}$ & Liquid $^{*}$ & $20^{* *}$ & 260 & nd & nd & nd & nd & 0.10 & 0.024 & nd & nd & nd \\
\hline $7 \mathrm{~A}$ & Liquid* & $30^{* *}$ & 290 & nd & nd & nd & nd & nd & 0.033 & nd & nd & nd \\
\hline $8 \mathrm{~A}$ & Liquid $^{*}$ & $40^{* *}$ & 110 & nd & nd & nd & nd & nd & nd & 0.19 & nd & nd \\
\hline $9 \mathrm{~A}$ & Solid & 14.2 & 400 & nd & nd & nd & nd & 5.3 & nd & nd & nd & 0.11 \\
\hline $10 \mathrm{~A}$ & Solid & 25.2 & 190 & nd & 0.14 & nd & nd & 7.9 & nd & nd & nd & 0.17 \\
\hline $11 \mathrm{~A}$ & Solid & 25.2 & - & \multicolumn{9}{|c|}{ TCLP analysis not performed } \\
\hline $12 \mathrm{~A}$ & Solid & 14.2 & 80 & nd & nd & nd & nd & 0.90 & nd & nd & nd & nd \\
\hline $13 \mathrm{~A}$ & Solid & 25.2 & 0 & nd & nd & nd & nd & 0.76 & nd & nd & nd & 0.13 \\
\hline \multicolumn{13}{|c|}{ Al LAW Simulant Samples } \\
\hline $14 \mathrm{~A}$ & Liquid & 20 & 170 & \multicolumn{9}{|c|}{ TCLP analysis not performed } \\
\hline $15 \mathrm{~A}$ & Liquid & 30 & 130 & nd & nd & nd & nd & nd & nd & nd & nd & nd \\
\hline $16 \mathrm{~A}$ & Solid & 14.0 & 120 & nd & nd & nd & nd & nd & nd & nd & nd & nd \\
\hline $17 \mathrm{~A}$ & Solid & 24.3 & 1030 & nd & nd & nd & nd & 0.07 & nd & nd & nd & nd \\
\hline \multicolumn{13}{|c|}{ Zr LAW Simulant Samples } \\
\hline $18 \mathrm{~A}$ & Liquid & 20 & 190 & nd & nd & nd & nd & nd & nd & nd & nd & nd \\
\hline $19 \mathrm{~A}$ & Liquid & 30 & 310 & nd & nd & nd & nd & nd & nd & nd & nd & nd \\
\hline $20 \mathrm{~A}$ & Liquid & 30 & 1260 & nd & nd & nd & nd & nd & nd & nd & nd & nd \\
\hline $21 \mathrm{~A}$ & Solid & 14.0 & 180 & nd & nd & nd & nd & nd & nd & nd & nd & nd \\
\hline $22 \mathrm{~A}$ & Solid & 24.3 & 470 & nd & nd & nd & nd & 0.17 & nd & nd & nd & nd \\
\hline $23 \mathrm{~A}$ & Solid & 14.0 & 30 & nd & nd & nd & 0.061 & nd & nd & nd & nd & nd \\
\hline
\end{tabular}


Table 7. Test Results for Portland Cement Based Grout Samples.

\begin{tabular}{|c|c|c|c|c|c|c|c|c|c|c|c|c|}
\hline \multirow[b]{2}{*}{$\begin{array}{c}\text { Sample } \\
\text { Num. }\end{array}$} & \multirow[b]{2}{*}{$\begin{array}{l}\text { Waste } \\
\text { Type }\end{array}$} & \multirow{2}{*}{$\begin{array}{l}\text { Waste } \\
\text { Load. } \\
\left(\mathrm{wt}^{\circ} \%\right)\end{array}$} & \multirow{2}{*}{$\begin{array}{l}\text { Comp. } \\
\text { Strength } \\
\text { (psig) }\end{array}$} & \multicolumn{9}{|c|}{ TCLP Results (mg/L) } \\
\hline & & & & $\mathrm{Ag}$ & As & $\mathrm{Ba}$ & $\mathrm{Cd}$ & $\mathrm{Cr}$ & $\mathrm{Hg}$ & $\mathrm{Ni}$ & $\mathrm{Pb}$ & $\mathrm{Se}$ \\
\hline \multicolumn{13}{|c|}{ Na LAW Simulant Samples } \\
\hline $1 \mathrm{~B}$ & Liquid $^{*}$ & $20^{* *}$ & 2840 & $\mathrm{nd}^{* * *}$ & nd & 0.71 & nd & nd & nd & nd & nd & nd \\
\hline $2 \mathrm{~B}$ & Liquid $^{*}$ & $30^{* *}$ & 4470 & nd & nd & 0.74 & nd & nd & nd & nd & nd & nd \\
\hline $3 \mathrm{~B}$ & Liquid* & $40^{* *}$ & 760 & nd & nd & 1.3 & nd & nd & nd & nd & nd & nd \\
\hline 4B & Liquid $^{*}$ & $62^{* *}$ & 100 & nd & nd & 1.9 & nd & nd & nd & nd & nd & nd \\
\hline $5 \mathrm{~B}$ & Solid & 14.2 & 3000 & na & na & na & nd & nd & nd & na & nd & na \\
\hline $6 \mathrm{~B}$ & Solid & 25.1 & 2360 & nd & nd & nd & nd & nd & nd & nd & nd & nd \\
\hline \multicolumn{13}{|c|}{ Al LAW Simulant Samples } \\
\hline $7 \mathrm{~B}$ & Liquid & 20 & 2540 & nd & nd & 0.82 & nd & nd & nd & nd & nd & nd \\
\hline $8 \mathrm{~B}$ & Liquid & 30 & 2830 & nd & nd & 0.83 & nd & nd & nd & nd & nd & nd \\
\hline 9B & Solid & 14.0 & 3450 & \multicolumn{9}{|c|}{ TCLP analysis not performed } \\
\hline $10 \mathrm{~B}$ & Solid & 24.3 & 2170 & \multicolumn{9}{|c|}{ TCLP analysis not performed } \\
\hline \multicolumn{13}{|c|}{ Zr LAW Simulant Samples } \\
\hline $11 \mathrm{~B}$ & Liquid & 20 & 4680 & nd & nd & 0.72 & nd & nd & nd & nd & nd & nd \\
\hline $12 \mathrm{~B}$ & Liquid & 30 & 3830 & nd & nd & 0.84 & nd & nd & nd & nd & nd & nd \\
\hline $13 \mathrm{~B}$ & Solid & 14.0 & 3520 & na & na & na & 0.02 & nd & nd & na & nd & na \\
\hline $14 \mathrm{~B}$ & Solid & 24.3 & 3430 & na & na & na & 0.26 & nd & nd & na & nd & na \\
\hline $\begin{array}{ll}* & \text { The Na } \\
& \text { LAW). } \\
* * & \text { The wt } \\
& \text { simula } \\
* * * & \text { nd }- \text { no } \\
& \text { na }- \text { sa }\end{array}$ & $\begin{array}{l}\text { AW simulant } \\
\text { waste loadin } \\
\text { etected in sa } \\
\text { le not analyz }\end{array}$ & $\begin{array}{l}\text { sed for this } \\
\text { f the doubl } \\
\text { for that el }\end{array}$ & $\begin{array}{l}\text { esting was } m \\
\text { concentratio } \\
\text { nent }\end{array}$ & $\begin{array}{l}\text { e doubl } \\
\text { Na LAW }\end{array}$ & imula & $\begin{array}{l}\text { wice th } \\
\text { s simpl }\end{array}$ & $\begin{array}{l}\text { concen } \\
\text { calculat }\end{array}$ & as $2 \mathrm{t}$ & s the $\mathrm{w}$ & $\begin{array}{l}\text { ions it } \\
\text { t fract }\end{array}$ & $\begin{array}{l}\text { he act } \\
\text { of the }\end{array}$ & \\
\hline
\end{tabular}




\subsection{Data Analysis/Interpretation}

PBC sample compression strengths were significantly lower than those of portland cement based grout samples with the same waste loadings. Note, however, that the PBC samples were prepared using a generalized recipe and procedure. The portland cement based grout samples were prepared using already optimized recipes and procedures. Specifically, the partial neutralization of the liquid waste simulant prior to dry powder addition was an optimization step used for portland cement based grout sample preparation. Including this same neutralization step for PBC sample preparation would likely also improve those respective compression strength (and possibly TCLP) results.

The two PBC samples made with increased $\mathrm{MgO}$ did demonstrate significantly improved compression strength results (Samples 5A over 1A, and 20A over 19A). These results support the notion that significant improvement to PBC wasteform properties could be achieved through process flowsheet optimization testing.

TCLP results for the portland cement based grout samples are also generally more favorable than those for the PBC samples. This is most evident in TCLP results for Na LAW simulant samples, as that waste type contains the greatest number and generally highest concentrations of hazardous metals. (Al LAW simulant contains no hazardous metals; the only hazardous metal contained in Zr LAW simulant is cadmium). For the portland cement based grout samples, barium is generally the only hazardous metal detected in the TCLP leachate, being at acceptable levels in all cases. Note that barium had not been added to any of the waste simulants, and is likely a contaminant in the BFS or one of the other additive cement powders. The exception was in the portland cement based grouts samples made with solid $\mathrm{Zr}$ LAW simulant, where cadmium was detected in the leachate. An unacceptable level of cadmium was found in the one sample made with $24.3 \mathrm{wt} \%$ solid $\mathrm{Zr}$ waste simulant.

For the PBC samples made with liquid Na LAW simulant, mercury is generally detected in the TCLP leachate, at acceptable concentrations. Unacceptable levels of chromium and selenium are generally detected in the leachate from the Na LAW solid simulant PBC samples.

One potential reason for the inferior $\mathrm{PBC}$ application test results is that although the phosphate bonding reactions can be initiated at ambient temperature, timely continuation of those reactions is likely dependent on the reaction heat generation. The 2-inch cube samples, with their respective high surface areas, may have been responsible for heat loss rates that inadvertently quenched the binding reactions during the initial curing period, despite the insulating efforts made. Similar makeups incorporating a higher grout mass per container vessel surface area could potentially result in a higher quality product.

The seemingly biggest drawback to $\mathrm{PBC}$ was in the sample preparation, with respect to the large deviation in required stir times for the different waste types and concentrations. Although this was a significant problem in this testing, it is probable that a desired stir time can be attained for any of the waste types through further development testing. However, it is also likely that a successful PBC flowsheet would be applicable to only a tight range of waste acidity/alkalinity, and any major deviation from that range would severely alter the required stir time and/or wasteform quality. 


\subsection{Conclusions}

The following conclusions have been drawn from this PBC application scoping study:

1. Acceptable wasteforms could be made using PBC technology for INTEC LAW immobilization.

2. As applied to INTEC LAW immobilization, portland cement based grout samples demonstrated compression strength and TCLP results superior to similar PBC sample results.

3. Significant further development/optimization work would be required before PBC technology could be successfully implemented for INTEC LAW immobilization.

4. A single PBC application "recipe" is not likely possible for all INTEC LAW immobilization. Required process flowsheet and/or procedure changes will accompany any significant change in waste acidity/alkalinity.

5. Although improvements on PBC immobilization technology application to INTEC LAW could certainly be made, there is no indication that even an optimized flowsheet and procedure for PBC application would result in wasteform compression strength or leachability properties superior to those attained using portland cement based grout.

6. PBC has the potential to immobilize INTEC LAWs at higher waste loadings than portland cement based grout.

7. Nitrate, and potentially other anions, will be readily leached from a PBC wasteform. 


\section{TANK FARM CLOSURE GROUTING DEVELOPMENT UPDATE}

A major component of the tank farm closure is the treatment and/or removal of the tank heels. Based on previous testing, tank heel displacement with grout and removal was determined favorable to direct heel grouting, with respect to both economics and wasteform quality ${ }^{6}$. It was also determined during the previous testing that the heel solutions should be diluted with water to no more than $12.5 \%$ original solution to prevent excessive chemical degradation of the grout.

Additional testing has been performed to determine an optimum grout formulation for this preferred heel displacement application. This "wasteform" application is unique in that no actual waste will be intentionally mixed into the grout. Conversely, this displacement grout will be subjected to potential chemical attack from the outside.

The goals of this testing are to determine the formulation for a grout which will cure adequately when directly exposed to the diluted acidic waste solution, experience minimal corrosion during that curing period, and possess a reasonable compressive strength ( $>100 \mathrm{psi})$ after solution removal and grout curing.

This tank heel displacement grout testing generally involved comparing the final compressive strengths of different grout formulations after periods of exposure to dilute tank farm simulant. During actual tank heel displacement with grout operations, the extent of grout contact with the acidic heel solution is expected to be relatively small (on a grout-to-waste solution mass basis), as compared to the significant grout sample exposure to heel simulant of this testing?. However, a grout capable of withstanding the conservative parameters of this testing more strongly assures success during actual application.

\subsection{Test Description}

Eight groups of six 2-inch cube grout samples each were prepared and immediately submerged in various tank farm waste simulant solutions for extended time periods. For each group, three samples were removed from the respective solution after approximately 30 days of curing, allowed to air dry for several hours, and subjected to compressive strength testing. This same sequence of removal from solution, drying, and strength testing was performed on the remaining three samples from each group after approximately 90 days of submersed curing. The grout formulation and the makeup of the submersion solution, for each sample group, are presented in Table 8. The chemical composition of the tank farm simulant referred to in the submersion solution makeups (prior to dilution) is presented in Table 9.

Grouts for Sample Groups 2, 3, 3b, 4, and 5 were made using a 1:3:1 weight ratio of Type I/II ordinary portland cement (OPC): blast furnace slag (BFS): Class F fly ash (FA). Grout for Sample Group 1 was made using a 1:1:1 weight ratio of the same ingredients. Grouts for Sample Groups 6 and 7 were both made using a large weight fraction of relatively non-reactive aggregate (sand). The recipes for both of these grouts (mortars) have been already used in the closures of actual radiation-contaminated DOE facilities. (Sample Group 6 grout was made using a recipe used for the INEEL WCF facility closure. Sample Group 7 grout was made using a recipe used for tank closures at Savannah River. 
Table 8. Test Grout Formulations and Makeups of Submersion Solutions.

\begin{tabular}{|c|c|c|c|c|c|c|c|c|}
\hline & \multicolumn{7}{|c|}{ Sample Group } & \multicolumn{1}{c|}{} \\
\cline { 2 - 9 } & $\mathbf{1}$ & $\mathbf{2}$ & $\mathbf{3}$ & $\mathbf{3 b}$ & $\mathbf{4}$ & $\mathbf{5}$ & $\mathbf{6}$ & $\mathbf{7}$ \\
\hline Grout Components & & & & & & & & \\
\hline Type I/II OPC (wt \%) & 23.4 & 14.0 & 14.7 & 14.0 & 14.0 & 13.3 & 9.0 & \\
\hline Type V OPC (wt \%) & & & & & & & & 34.6 \\
\hline BFS (wt \%) & 23.4 & 42.1 & 44.2 & 42.1 & 42.1 & 40.0 & & 5.3 \\
\hline FA (wt \%) & 23.4 & 14.0 & 14.7 & 14.0 & 14.0 & 13.3 & 17.7 & \\
\hline Water (wt \%) & 29.8 & 29.8 & 26.4 & 29.8 & 29.8 & 33.3 & 14.0 & 18.4 \\
\hline Sand (wt \%) & & & & & & & 59.3 & 41.6 \\
\hline $\begin{array}{c}\text { Submersion Solution } \\
\text { Components }\end{array}$ & & & & & & & & \\
\hline $\begin{array}{c}\text { Tank Farm Waste Simulant } \\
\text { (wt \%) }\end{array}$ & 12.5 & 12.5 & & & 12.5 & 12.5 & 12.5 & 12.5 \\
\hline
\end{tabular}

Table 9. Tank Farm Simulant Chemical Composition (Prior to Dilution)

\begin{tabular}{|c|c|}
\hline Chemical Species & Concentration (Molar) \\
\hline $\mathrm{H}+$ & $1.57 \mathrm{E}+00$ \\
\hline $\mathrm{Al}$ & $6.39 \mathrm{E}-01$ \\
\hline $\mathrm{B}$ & $1.80 \mathrm{E}-02$ \\
\hline $\mathrm{Ca}$ & $5.27 \mathrm{E}-02$ \\
\hline $\mathrm{Cl}$ & $2.87 \mathrm{E}-02$ \\
\hline $\mathrm{Cs}$ & $1.05 \mathrm{E}-05$ \\
\hline $\mathrm{F}$ & $6.89 \mathrm{E}-02$ \\
\hline $\mathrm{Fe}$ & $2.20 \mathrm{E}-02$ \\
\hline $\mathrm{Mn}$ & $1.22 \mathrm{E}-02$ \\
\hline $\mathrm{Mo}$ & $6.37 \mathrm{E}-04$ \\
\hline $\mathrm{K}$ & $2.04 \mathrm{E}-01$ \\
\hline $\mathrm{Sr}$ & $5.10 \mathrm{E}-06$ \\
\hline $\mathrm{Na}^{2}$ & $1.89 \mathrm{E}+00$ \\
\hline $\mathrm{NO}_{3}$ & $5.17 \mathrm{E}+00$ \\
\hline $\mathrm{PO}_{4}$ & $1.40 \mathrm{E}-02$ \\
\hline $\mathrm{SO}_{4}$ & $5.04 \mathrm{E}-02$ \\
\hline
\end{tabular}


Submersion solution used in most cases consisted of $12.5 \%$ (by volume) non-toxic tank farm simulant, and $87.5 \%$ water. Exceptions to this were: groups 3 and $3 \mathrm{~b}$ samples were submerged in water as a base case; group 4 samples were submerged in a simulant of tank farm waste diluted with $2.2 \underline{\mathrm{M}}$ aluminum nitrate and water, to simulate using such a solution for tank "washout" prior to heel displacement.

For the initial sample preparations (chronologically, Sample Groups 3, 2, and 5), the 2-inch cube plastic molds were set inside individual plastic containers, and the molds were filled with the respective submersion solution until they overflowed inside the respective plastic containers. The grout ingredients were mixed separately in batches, and the fluid grout was poured into the molds, displacing solution into the containers. Enough grout was poured into each mold to fill it. Enough solution was used (approximately $380 \mathrm{ml}$ per sample) so that when each mold was filled with grout, the solution completely covered the sample.

Preparation of the initial Sample Group 3 left significant voids in the samples as a result of pouring the relatively thick grout into the small, solution-filled molds. As a result, the water-to-solids mass ratio (w/s) of 0.359 used for Sample Group 3 was increased to 0.425 for Sample Groups 1, 2, 3b, and 4. Group $3 \mathrm{~b}$ testing was identical to that of Group 3, except for the increased w/s. Group 5 testing was identical to that of Groups 3 and $3 b$, except for a further increase of w/s to 0.50 . The water contents for Sample Groups 6 and 7 grout preparations were dictated by the recipes for those grouts, as these are both pre-developed formulations. The mixed grout for Sample Group 7 was noticeably the most fluid of all grouts made in this testing.

Continued difficulties experienced with sample preparation by simulant solution displacement in the molds led to a change in that procedure. Samples for groups 3, 2, and 5 had been prepared using the solution displacement method. Sample preparation for all remaining groups differed from above in that the sample molds were filled with the respective batch-mixed grouts outside the submersion containers, and then immediately submerged in the liquid inside them. To prevent voids in the samples, the molds were continually "tapped" during the grout-filling step.

Just prior to compressive strength testing, all group samples were visually inspected for evidence of corrosion. Sample weights, before and after curing, were also taken in most cases. Although informative, results from these steps are qualitative as submersion curing of grout effects the ultimate sample water content, and chemical reactions do occur at the grout/solution interface. Thus, accurate sample weight change determinations are not possible. Major sample weight decreases $(>5 \%)$ do, however, flag a potentially significant corrosion problem.

\subsection{Test Results}

Compressive strength and qualitative corrosion results for each Sample Group are presented in Table 10. Unless otherwise indicated, compressive strength results presented are the average of those obtained from three separate samples from the respective test group. Also indicated is the maximum deviation of either of the three sample strengths from the average. 
Table 10. Grout Sample Compressive Strength and Corrosion Results

\begin{tabular}{|c|c|c|c|c|}
\hline $\begin{array}{l}\text { Sample } \\
\text { Group }\end{array}$ & $\begin{array}{l}\text { Average 30-Day } \\
\text { Compressive Strength } \\
\text { (psi) }\end{array}$ & $\begin{array}{c}\text { Average 90-Day } \\
\text { Compressive Strength } \\
\text { (psi) }\end{array}$ & $\begin{array}{c}\text { 30-Day Extent of } \\
\text { Corrosion } \\
\text { (Qualitative) }\end{array}$ & $\begin{array}{c}\text { 90-Day Extent of } \\
\text { Corrosion } \\
\text { (Qualitative) }\end{array}$ \\
\hline 1 & $\begin{array}{c}620 \\
+/-3 \%\end{array}$ & $\begin{array}{c}610 \\
+/-4 \%\end{array}$ & $\begin{array}{c}\text { Minimal } \\
\text { No noticeable weight } \\
\text { change }\end{array}$ & $\begin{array}{c}\text { Minimal } \\
\text { No noticeable weight } \\
\text { change }\end{array}$ \\
\hline 2 & NA* & $\begin{array}{c}770 \\
+/-10 \%\end{array}$ & $\begin{array}{c}\text { Minimal } \\
\text { No noticeable weight } \\
\text { change }\end{array}$ & $\begin{array}{c}\text { Minimal } \\
\text { No noticeable weight } \\
\text { change }\end{array}$ \\
\hline 3 & $\begin{array}{c}1080 \\
+/-22 \%\end{array}$ & $\begin{array}{c}800 \\
+/-21 \%\end{array}$ & $\begin{array}{c}\text { Minimal } \\
\text { No noticeable weight } \\
\text { change }\end{array}$ & $\begin{array}{c}\text { Minimal } \\
\text { No noticeable weight } \\
\text { change }\end{array}$ \\
\hline $3-b$ & $\begin{array}{c}650 \\
+/-24 \%\end{array}$ & $\begin{array}{c}580 \\
+/-15 \%\end{array}$ & $\begin{array}{c}\text { Minimal } \\
\text { No noticeable weight } \\
\text { change }\end{array}$ & $\begin{array}{c}\text { Minimal } \\
\text { No noticeable weight } \\
\text { change }\end{array}$ \\
\hline 4 & $\begin{array}{c}530 \\
+/-13 \%\end{array}$ & $\begin{array}{c}660 \\
+/-13 \%\end{array}$ & $\begin{array}{c}\text { Moderate } \\
\text { Approx. } 10 \% \text { sample } \\
\text { weight reduction }\end{array}$ & $\begin{array}{c}\text { Moderate } \\
\text { Over } 10 \% \text { sample } \\
\text { weight reduction }\end{array}$ \\
\hline 5 & $\begin{array}{c}350 \\
+/-24 \%\end{array}$ & $\begin{array}{c}300 \\
+/-10 \%\end{array}$ & $\begin{array}{l}\text { High } \\
\text { Approx. } 15 \% \text { sample } \\
\text { weight reduction }\end{array}$ & $\begin{array}{c}\text { Extreme } \\
\text { Approx. 25\% sample } \\
\text { weight reduction }\end{array}$ \\
\hline 6 & $\begin{array}{c}680 \\
+/-3 \%\end{array}$ & $\begin{array}{l}1290 * * \\
+/-18 \%\end{array}$ & $\begin{array}{c}\text { Minimal } \\
\text { No noticeable weight } \\
\text { change }\end{array}$ & $\begin{array}{c}\text { Minimal } \\
\text { No noticeable weight } \\
\text { change }\end{array}$ \\
\hline 7 & $\begin{array}{c}1140 \\
+/-21 \%\end{array}$ & $\begin{array}{l}1480 * * \\
+/-15 \%\end{array}$ & $\begin{array}{c}\text { Minimal } \\
\text { No noticeable weight } \\
\text { change }\end{array}$ & $\begin{array}{c}\text { Minimal } \\
\text { No noticeable weight } \\
\text { change }\end{array}$ \\
\hline \multicolumn{5}{|c|}{$\begin{array}{l}\text { * NA- results obtained are in error } \\
\text { ** 90-day compressive strength averages for sample groups } 6 \text { and } 7 \text { were calculated using six samples each, and throwing out } \\
\text { the low value. In both cases, the low value exceeded the } 500 \text {-psi NRC minimum limit. }\end{array}$} \\
\hline
\end{tabular}

The cured cubes from Sample Groups 1, 2, 3, 3b, 6, and 7 (following the drying period, but prior to the compressive strength test) were all hard with no visual evidence of corrosion. The cured cubes from Sample Groups 4 and 5 did show significant signs of corrosion. After 30 days of curing, the Sample Group 4 cubes (cured while submerged in dilute aluminum nitrate/waste simulant solution) were all soft, at the exposed surface. After 90 days of submersion curing, group 4 samples were soft at the exposed side to a depth of approximately $1 / 4$ inch. All other cube sides were course and rough (as compared to samples from other groups). All sample sides had receded from the mold walls approximately 1/16 inch. The Sample Group 5 cubes (which had used excess water in the grout makeup) were all layered from top to bottom, with the top layers having no strength and being unattached to the remaining monolithic samples. After 30 days of submersion curing, the unattached top layer was over $1 / 4$-inch thick. After 90 days of submersion curing, the unattached top layer was over $1 / 2$-inch thick. 


\subsection{Results Discussion}

As expected, compressive strength results decreased as more water was included in the grout makeup for the base-case tests (Sample Groups 3 and 3b). Not expected was the base-case test where the samples were submerged in water (Sample Group 3b) having slightly lower compressive strength values than did samples which had the same water content and chemical makeup (Sample Group 2) but were cured while submerged in dilute acidic tank farm simulant. This information indicates that the accuracy and repeatability of these tests is not high, and that the effects of the dilute waste solution on the physical properties of the grouts are minimal.

Seemingly more important are the noticeable detrimental effects of adding too much water to the grout mix (Sample Group 5) and exposing the curing grout to aluminum nitrate solution (Sample Group 4).

\subsection{Conclusions}

Conclusions from this testing are as follows:

1. Both the 1:1:1 and 1:3:1 mass ratio grout blends of OPC:BFS:FA (Sample Groups 1 and 2, respectively) will successfully cure, and suffer minimal corrosion damage, while submerged in the dilute (12.5 volume \%) tank farm waste solution, provided the grout is prepared using a w/s mass ratio of less than 0.425 . The resulting average compression strengths of these grouts are both acceptable for this application.

2. Increasing the grout mix water content above a $0.425 \mathrm{w} / \mathrm{s}$ mass ratio will have detrimental effects on the physical properties of the final monolith. Conversely, improved grout compressive strength and other physical properties will be realized by reducing the grout water content to as low as possible while still maintaining adequate fluidity.

3. Using aluminum nitrate solution instead of water as a heel diluent will result in grout decomposition at the solid-liquid interface. It is essential that if this method of grout displacement is used, the waste solution must be removed promptly to assure minimal destruction of the grout and to prevent solids precipitation from the liquid waste.

4. The WCF cell fill grout and the Savannah River "reducing grout" (Sample Groups 6 and 7, respectively) are both made with a large mass fraction of inexpensive aggregate (sand). Test results show both of these grouts to have compressive strengths superior to those of all other grouts tested, and to be acceptable for this submerged curing/tank heel removal application. The Group 7 (SR reducing grout) test samples had compressive strengths slightly superior to those obtained for Group 6 samples. 


\subsection{Recommendations}

Recommendations from this testing are to employ the Savannah River reducing grout for the tank closure/dilute waste solution displacement application because:

1. This formulation provides the most "self-leveling" (highest slump) grout of all tested.

2. The "advertised" reducing nature of this grout will likely cause any contacted hazardous cations to be less soluble than will the other grouts.

3. The compression strength of this grout exceeds those of all others tested.

4. The proposed application is very similar to the Savannah River tank closure, for which this grout has already been accepted and used.

5. Using this grout formulation, with a large mass fraction being sand, will likely be less costly than will using most others tested. 


\section{CEMENTITIOUS IMMOBILIZATION OF STRONGLY ACIDIC, NITRATE SALT SOLUTIONS}

Nuclear fuel reprocessing activities at the Idaho Nuclear Technology and Engineering Center (INTEC) on the Idaho National Engineering and Environmental Laboratory (INEEL) have left a legacy of highly acidic, alkali nitrate waste solutions stored in stainless steel tanks that do not meet modern environmental standards. Continuing waste treatment and supporting operations generate additional acidic waste that will require immobilization prior to disposal. Both of these radioactive solutions contain significant quantities of characteristically hazardous heavy metals and highly-concentrated alkali nitrate salts. Conventional thought is that acidic solution must be rendered basic prior to being stabilized in portland cement grouts, while trying to avoid formation of sludges that can result when solutions are driven basic. So far, "basic waste" grouting is proven to be the more stable waste form, however there are two unfavorable factors that have led to researching a method of directly grouting the acidic waste without pretreatment to a basic solution. These two characteristics are (1) a three day time element along with an increase in process complexity in driving the waste solution to basic and (2) an increase in final waste form volume from original waste solution volume. To try and solve these unfavorable conditions, a waste form made by mixing grout forming powders directly with the acidic waste solution after the solution $\mathrm{pH}$ was brought up to 0.7 was developed and tested to ensure the long term stability. Stability tests recommended in the conservative Nuclear Regulatory Commission (NRC) Technical Position on Waste Form were followed. This "acidic" grout has exceeded requirements for 28-day compressive strength and met requirements for nitrate leachability. Long-term immersion tests and thermal cycling weakened the waste form and caused visible cracking and/or spalling. In the case of immersion, a larger deviation was created from the 28-day compressive strength than allowed. One other important waste form qualification test, not specified by the NRC, is the toxic characteristic leach procedure (TCLP) to assess the retention of toxic elements. The "acidic" waste form passed this test.

Dimensional stability was determined using prisms 0.75 -mm square x 250 -mm long by measuring change in length per original length over time. The length change data indicates the grout volume change while curing. ${ }^{8}$

These results are promising, however, the formulation needs improvement and it is felt that endurance strengths can be improved with a better understanding of the acidic chemistry. It is noted that despite the failure of these two NRC suggested criteria, acidic grout does allow for disposal of INTEC acidic waste into NRC Low-Level Class A waste or non-NRC repositories.

\subsection{Materials and Methods}

This section describes the simulated waste constituents and the type of grout forming powderscement (ordinary Portland cement Type I/II), slag (blast-furnace slag), and fly ash (pulverized-coal fuel ash, Type F) used to immobilize the acidic waste solutions. Some discussion about the reasons for choosing these three grout formers is also provided. 


\subsection{Waste Solution Simulant}

A simulant was prepared to match the future composition of the highly acidic, alkali nitrate waste solutions stored in stainless steel tanks located at the INEEL. Two types of simulant were prepared; (1) a simulant without characteristically toxic metal salts and (2) a hazardous inoculant with the elements Ag, $\mathrm{As}, \mathrm{Ba}, \mathrm{Cd}, \mathrm{Cr}, \mathrm{Hg}, \mathrm{Ni}, \mathrm{Pb}$, and $\mathrm{Se}$. Grout formulations were developed using the non-toxic simulant and validated with an inoculated simulant. Table 11 shows the concentrations of the major contributing species and the stock chemical compounds used to achieve those molarities. The nominal nitrate content is 28 grams per 100 grams of solution ( $\mathrm{wt} \%)$. The $\mathrm{pH}$ is less than one.

Table 11. Waste Simulant Compositions. Concentrations of major species in the highly acidic, alkali nitrate waste solutions stored at INEEL.

\begin{tabular}{|c|c|c|}
\hline Species & $\begin{array}{c}\text { Species Concentration } \\
(\mathrm{mg} / \mathrm{L})\end{array}$ & $\begin{array}{c}\text { Species Molarity } \\
(\mathrm{M})\end{array}$ \\
\hline $\mathrm{H}$ & $1.54 \mathrm{~N}$ & $1.54 \mathrm{E}+00$ \\
\hline $\mathrm{Al}$ & $1.82 \mathrm{E}+04$ & $6.74 \mathrm{E}-01$ \\
\hline B & $2.05 \mathrm{E}+02$ & $1.89 \mathrm{E}-02$ \\
\hline $\mathrm{Ca}$ & $2.24 \mathrm{E}+03$ & $5.58 \mathrm{E}-02$ \\
\hline $\mathrm{Cl}$ & $1.09 \mathrm{E}+03$ & $3.06 \mathrm{E}-02$ \\
\hline $\mathrm{Cs}$ & $1.54 \mathrm{E}-04$ & $1.16 \mathrm{E}-09$ \\
\hline $\mathrm{F}$ & $1.38 \mathrm{E}+03$ & $7.26 \mathrm{E}-02$ \\
\hline $\mathrm{Fe}$ & $1.30 \mathrm{E}+03$ & $2.32 \mathrm{E}-02$ \\
\hline $\mathrm{Mn}$ & $9.25 \mathrm{E}+02$ & $1.68 \mathrm{E}-02$ \\
\hline Mo & $7.10 \mathrm{E}+01$ & $7.36 \mathrm{E}-04$ \\
\hline $\mathrm{Ni}$ & $9.90 \mathrm{E}+01$ & $1.68 \mathrm{E}-03$ \\
\hline $\mathrm{NO}_{3}$ & $3.31 \mathrm{E}+05$ & $5.34 \mathrm{E}+00$ \\
\hline $\mathrm{PO}_{4}$ & $0.00 \mathrm{E}+00$ & $0.00 \mathrm{E}+00$ \\
\hline K & $8.45 \mathrm{E}+03$ & $2.16 \mathrm{E}-01$ \\
\hline $\mathrm{Na}$ & $5.07 \mathrm{E}+04$ & $2.20 \mathrm{E}+00$ \\
\hline $\mathrm{SO}_{4}$ & $5.05 \mathrm{E}+03$ & $5.26 \mathrm{E}-02$ \\
\hline $\mathrm{Zr}$ & $9.60 \mathrm{E}+01$ & $1.05 \mathrm{E}-03$ \\
\hline $\mathrm{As}^{\mathrm{a}}$ & $5.00 \mathrm{E}+00$ & $6.22 \mathrm{E}-05$ \\
\hline $\mathrm{Ba}^{\mathrm{a}}$ & $9.00 \mathrm{E}+00$ & $6.42 \mathrm{E}-05$ \\
\hline $\mathrm{Cd}^{\mathrm{a}}$ & $3.30 \mathrm{E}+02$ & $2.94 \mathrm{E}-03$ \\
\hline $\mathrm{Cr}^{\mathrm{a}}$ & $2.03 \mathrm{E}+02$ & $3.90 \mathrm{E}-03$ \\
\hline $\mathrm{Pb}^{\mathrm{a}}$ & $3.05 \mathrm{E}+02$ & $1.47 \mathrm{E}-03$ \\
\hline $\mathrm{Hg}^{\mathrm{a}}$ & $2.30 \mathrm{E}+02$ & $1.15 \mathrm{E}-03$ \\
\hline $\mathrm{Se}^{\mathrm{a}}$ & $2.00 \mathrm{E}+00$ & $3.00 \mathrm{E}-05$ \\
\hline $\mathrm{Ag}^{\mathrm{a}}$ & $3.00 \mathrm{E}+00$ & $2.42 \mathrm{E}-05$ \\
\hline
\end{tabular}

a. Hazardous elements included only in inoculant. 


\subsection{Grout Forming Powders}

\subsubsection{Portland Cement, Type I/II}

Type I/II cement was used because it is the most common and readily accessible type of cement available. The beneficial characteristics that the other types of cement ( i.e. quick setting, sulfate resistant, or low heat of hydration) were viewed as unnecessary for solidification of INTEC acidic waste. Ash Grove Cement Company located in Inkom, Idaho supplied the Type I/II Portland cement used in this study.

\subsubsection{Blast-Furnace Slag}

The addition of ground blast furnace slag provides the cement mixture with reduced permeability to water and heavy metals. This may be achieved by a number of methods. The sulfides in the slag precipitates the heavy metals into less leachable metal sulfide species. The slag gives the cement form a dense microstructure of hydrated cement paste, thus decreasing the porosity of the cured grout and inhibiting mobility of encapsulated substances. ${ }^{9}$ It has been shown that slag cements inhibit the leachability of nitrates. ${ }^{10}$ Another advantage gained by using slag is the reduction in the water demand while improving workability. ${ }^{9}$ For this study Blue Circle Cement, Inc located in Sparrows Point, Maryland supplied the blast-furnace slag.

\subsubsection{Fly Ash, Type F}

Coupled with slag, fly ash improves the workability of the grout mixture while reducing the water needed to form a mixable paste. ${ }^{9}$ It is thought that this benefit is achieved by a "ball-bearing effect" that the spherical fly ash particles have on the rest of the grout mixture. Fly ash may become a less important additive as the waste loadings increase because the strength gained from fly ash is only realized when the pore water is highly basic. Higher waste loadings may decrease that basicity and thereby not activate the fly ash to contribute to the cement paste structure. This will be investigated in future studies. Valley Ready Mix, Inc. of Idaho Falls, Idaho supplied the fly ash taken from the Jim Bridger Power Plant in Wyoming.

\subsection{Grout Mixing}

Grout formers, in a ratio of 53\% slag, $40 \%$ cement, and $7 \%$ fly ash, are stirred one at a time into the highly acidic, alkali nitrate waste solution simulant, after addition of sodium hydroxide to bring the $\mathrm{pH}$ to approximately 0.7 . A previous scoping study showed two "trends" that were followed in this study: 1) an empirical observation that the ratios of grout formers should approximately equal the above ratio and 2) that a slight advantage in 28 -day compressive strength is gained by sequentially adding the slag, then cement, and finally fly ash. The resultant slurry was mixed for five minutes with a KitchenAid planetary mixer after each powder addition. After slag and simulant are mixed, the slurry thickens in a quick reaction presumed to be precipitating elements. Hydrogen sulfide gas is a byproduct of the reaction between the slag and waste simulant; so mixing was done in a fume hood. After five minutes of stirring, the slurry thinned to a paste and the cement powder was added. After five more minutes fly ash was added. A plastic spatula was used periodically to scrape down unmixed powders and grout mixture from the sides of the bowl during mixing. A test series for a grout recipe consisted of nine 2-inch cubes providing three cubes each for the 28-day cure, the 90-day immersion, and the thermal cycle crush strength tests. The cubes were cured in three ELE International cube mold (model CT-60). High-vacuum silicon grease was spread to seal the joints of the molds and a vegetable oil-based spray was applied to the 
surfaces to prevent sticking; Parafilm was overlaid to cover the top of the molds while the cubes cured to inhibit moisture loss. On average, the cubes required two days set time in the molds until they were sufficiently hardened to allow removal the cubes were placement in sealed plastic bags to finish the 28-day cure period at room temperature. There was no humidity regulation other than a sealed plastic bag and the temperature was not monitored. At the end of 28 days, the cubes were compression tested and then, if hazardous metals were added to the waste simulant, pieces of the cubes were leach tested (TCLP) to assess the hazardous element retention in grout.

\subsection{Performance Tests}

This section discusses the NRC guidelines on grout performance taken from the report titled Technical Position on Waste Form. The guideline report states compressive strengths for grouted waste forms after three different endurance tests; 28-day cure, thermal cycling, and water immersion. The report also suggests that the grouted waste form should be subject to leach testing. The two tests used for this purpose were ANSI/ANS 16.1 leach test, which applies to nitrates and the TCLP.

\subsubsection{NRC Position on Grout Performance}

Three areas of compressive testing were completed on the grout waste forms as suggested by the NRC. Grout waste forms must pass all three tests for disposal in a NRC landfill. The suggested requirements are more conservative than other waste acceptance criteria for other repositories. The NRC guidelines were used so that any error would be on the conservative side. The three time requirements for the compressive testing are after 28 days of normal curing, 30 thermal cycles from -40 to $60{ }^{\circ} \mathrm{C}$, and 90 days of immersion in water.

4.5.1.1 28-Day Cure - The grout waste form must have a minimum of 500 psi in mean compressive strength. Grouted waste with this amount of strength assumed to maintain integrity over time and stay structurally sound.

4.5.1.2 Thermal Cycling-There must be no visible defects after 30 thermal cycles; no cracking, spalling, or bulk disintegration. The cycles must range from -40 to $60{ }^{\circ} \mathrm{C}$ and pause long enough that temperature equilibrium is reached at each extreme. After passing visual inspection, the grouted waste form must have a compressive strength that is greater than or equal to $500 \mathrm{psi}$.

4.5.1.3 Immersion-Following a 28-day cure period and subsequent completion of 90-day water immersion, the grouted waste form must retain at least 75 percent of the 28-day cure compressive strength. If the strength is less than the 75 percent, but greater than $500 \mathrm{psi}$, the test should be extended to 120,150 , and 180 days to show that the strength does not decline below 500 psi. No extended tests beyond 90 days were done in this study.

\subsubsection{Compressive Strength}

All cubes were cured, in sealed plastic bags for a minimum of 28-days prior to crushing, then subjecting them to thermal cycling, the 90 -day water immersion, or compression testing.

A Tinius Olsen hydraulic compression machine was used to strength test the two-inch cubes. Data for compression rate and pressure as a function of time was controlled and recorded by a software program. The cube left in the sealed plastic bag and placed on the platform to be tested. 


\subsubsection{Nitrate Leach Test}

The abbreviated experimental procedure outlined in the ANSI/ANS 16.1 was followed to determine a leachability index for nitrate leaching. Nitrate leach testing was done using grout and waste simulant formed into frustum having a top and bottom diameter of 0.75 and 0.73 inch with a surface area of $24 \mathrm{~cm}^{2}$. The frustum design aided in the removal of the grout from machined polypropylene molds. A plastic wire tie was inserted into the paste for a hanger to suspend the frustum from a bottle lid during leach testing in deionized water. Three frusta were made for each different grout formulation. Once hardened for about 2 days, the frusta were removed from the molds and placed in sealed plastic bags to finish the 28-day cure period. Then the cured frustum, using the imbedded plastic wire tie, were secured through a hole drilled in a $250 \mathrm{ml}$ polypropylene bottle lid. The bottle held $240 \mathrm{ml}$ of deionized water as prescribed by the ANSI/ANS 16.1 leach test. Note that the leach test was modified so that it could be accomplished during a four-day workweek. Since the leachability index is calculated from the time dependant equations, the change does not affect the outcome. Therefore, the mechanism for diffusion remains constant.

\subsubsection{TCLP}

Since this was a scoping study, most tests were made with non-hazardous simulants. Thus, only a few mixtures were prepared with hazardous inoculants for TCLP testing. An external laboratory performed the TCLP on grouted waste form pieces produced from compression tests. The laboratory analyzed for cadmium, chromium, lead, mercury, and nickel.

\subsubsection{Dimensional Stability}

A Two-gang Prism Mold (supplied by ELE International) to produce a 0.75-mm square x 250-mm long prism was used along with the CT-384 series Length Comparator (supplied by ELE International) to indicate length changes. Following prism formation procedures outlined in the ASTM C 490-96 Standard Practice, grout gel is smoothed into the vegetable-oiled mold. When the grouted waste form is firm enough to be handled, the prism is removed and Parafilm is heat shrinked to the surface to help prevent moisture loss. The wrapped prism was then placed on a grill above a bath of water to allow humid condition curing. The prism has a large surface area to internal volume ratio and can loose moisture readily causing inaccurate results should the prism dry. The prism was measured with the Length Comparator at the time of removal from the mold and again at 28 days of curing. The dimensional stability is reported as a length change over total length (percent). The equations and procedures followed in the study are from ASTM C 490-96. ${ }^{11}$

\subsection{Results and Discussion}

\subsubsection{Compressive Strength}

The grouted waste form shows high compressive strengths at 28 days of curing even though the waste solution was not preconditioned to an basic state. However, compression tests performed after thermal cycling and immersion show that further development is needed to have the grouted waste form meet NRC recommendations. Figure 1 shows the relationship between the waste weight percent and the nominal compressive strength of grout forms after the three compressive tests. 


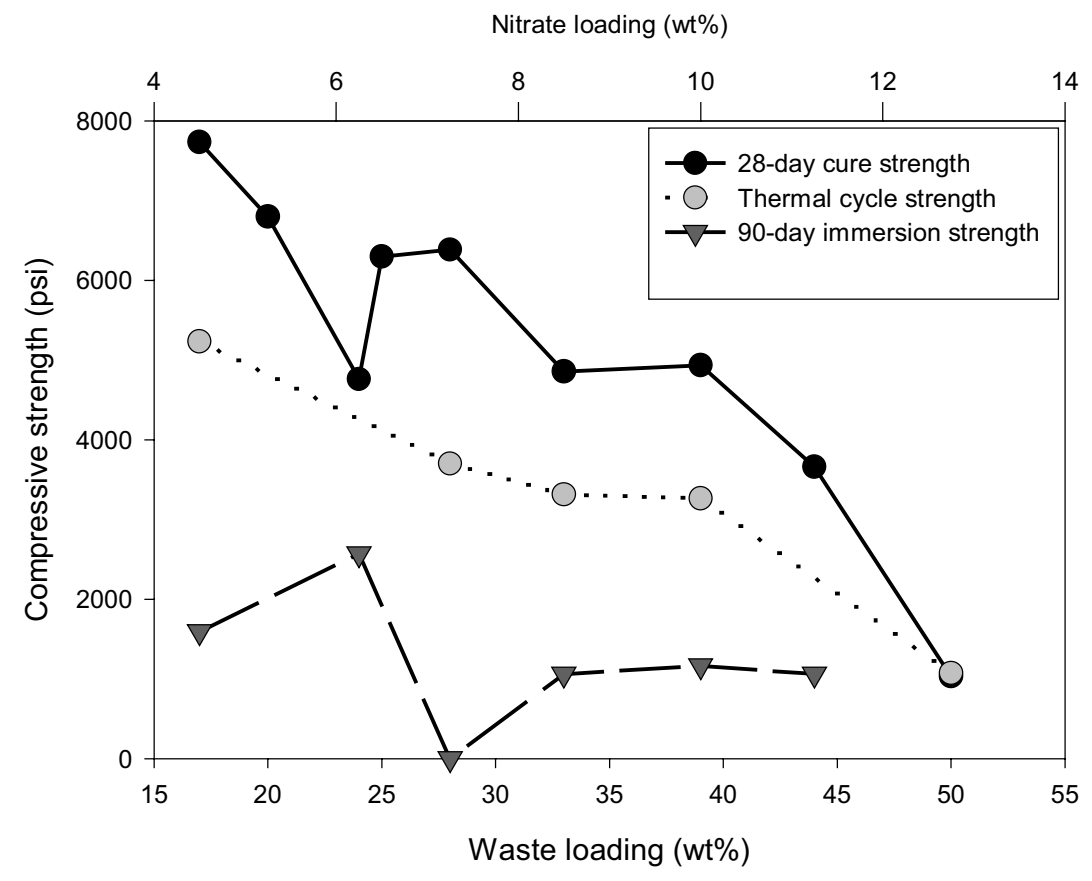

Figure 1. Influence of waste loading on compressive strength 
As mentioned before, the weight percentages of slag, cement, and fly ash were approximately 53, 40, and 7, respectively. This is a general trend observed by looking at compression strengths of grouted waste forms in previous scoping. The trend shows that a higher ratio of slag and a lower ratio of fly ash should be used as waste weight percent increase. This study does not propose an absolute upper limit in slag but it should be close to $53 \mathrm{wt} \%$. The trend of reducing fly ash as the acid waste loadings increase, seems to correlate with the understanding that high alkaline pore water is necessary to activate the cementitious properties of the ash.

All grout waste forms passed the NRC requirements for thermal cycling. It should be noted however, that cubes curing in a low humidity environment can exhibit hairline cracks. Water leaving the outer pore spaces of the cube appears to be the reason why cubes shrink and crack. Cubes cured in a plastic bag do not suffer from this shrinkage or crack.

The dip in compressive strength at 24 percent waste loading for 28-day cure strength may be explained by a slightly higher $\mathrm{pH}$ of the waste solution; 1.3 instead of the usual 0.7 . This was the only difference in the mixing procedure and may account for the variance in the trend shown in Figure 1. However, the strength after the 90-day water immersion increased when compared to the trend. This requires further study when the basics of acid chemistry in the formation of grout is studied.

None of the grouted waste forms passed immersion test requirements. Figure 1 shows a grouted waste form with 27 percent waste loading having zero compressive strength. The waste form had severe cracking and corners that had sloughed off destroying the three cubes integrity. It is not known why, at this waste loading, the grouted waste form completely failed. Grouts formed with high slag and low fly ash weight percents survived the immersion test without cracks and were strong enough to exceed 500 psi. For the same reason that extremely dry conditions cause the grouted waste forms to shrink, highly moist conditions allow for water to enter the pore spaces and exert enough hydraulic force to crack the cubes. Again, all cracking was in the outer portion of the cube, which supports this conclusion. With an increased understanding of the basic chemistry between the waste solution and grout formers, it is felt that a solution can be found.

\subsubsection{Nitrate Leach Test}

The nitrate leach index for acidic grout, calculated by following the procedure in the ANSI 16.1 leach rate test, matched levels reported in the literature. ${ }^{12}$ Table 12 shows leach indexes for three grout samples. To pass the leach test, a species must have a leachability index greater than six. The leachability index is explained with two numbers; confidence range and correlation coefficient. Repeatable index values should fall within the confidence range. Nitrate loading in weight percent and values for a denitrated waste grout form are also listed for comparative purposes. Again, data is limited for acid grout. As expected, the leachability index decreases as the waste loading increases. In comparison to other reported grouted waste forms, there is no advantage of direct grouting on the nitrate anion leachability, or disadvantage.

\subsubsection{TCLP}

Grouted waste forms with waste loadings of 25, 39, and 44 percent passed TCLP analysis. Table 13 shows the results of the three grouted waste forms. To compare, the levels for the Environmental Protection Agency (EPA) and Universal Treatment Standards (UTS) are shown. 
Table 12. Nitrate leachability index versus waste weight percent. The leach indexes for acidic grout are similar to values obtained for grouted waste forms using preconditioned waste solutions.

\begin{tabular}{ccc}
\hline $\begin{array}{c}\text { Waste loading } \\
(\mathrm{wt} \%)\end{array}$ & $\begin{array}{c}\text { Nitrate loading } \\
(\mathrm{wt} \%)\end{array}$ & $\begin{array}{c}\text { Leachability index } \\
\text { Confidence range } \\
(99 \%)\end{array}$ \\
\hline 17 & 4.7 & $8.0-9.3$ \\
39 & 11 & $7.1-7.9$ \\
$30^{\mathrm{a}}$ & 14 & $7.5-9.4$ \\
\hline
\end{tabular}

a. A grout form containing calcined acidic waste with 46 percent nitrate content.

Table 13. TCLP Results. Grouted waste forms pass TCLP at three varied waste loadings.

\begin{tabular}{ccccccc}
\hline \multicolumn{3}{c}{$\begin{array}{c}\text { Grouted Waste Samples } \\
(\text { wt\% waste) }\end{array}$} & & & & \\
\cline { 2 - 4 } & $\begin{array}{c}25 \\
(\mathrm{ppm})\end{array}$ & $\begin{array}{c}39 \\
(\mathrm{ppm})\end{array}$ & $\begin{array}{c}44 \\
(\mathrm{ppm})\end{array}$ & $\begin{array}{c}\text { EPA Limit } \\
(\mathrm{ppm})\end{array}$ & $\begin{array}{c}\text { UTS Limit } \\
(\mathrm{ppm})\end{array}$ & $\begin{array}{c}\text { Est. Instrument } \\
\text { Detection } \\
\text { Limit }\end{array}$ \\
\hline $\mathrm{Ag}$ & $\begin{array}{c}\text { Not } \\
\mathrm{Tested}\end{array}$ & $<\mathrm{DL}$ & $<\mathrm{DL}$ & 5 & 0.3 & 0.054 \\
$\mathrm{Cd}$ & $<\mathrm{DL}$ & 0.17 & $<\mathrm{DL}$ & 1 & 0.19 & 0.13 \\
$\mathrm{Cr}$ & $<\mathrm{DL}$ & 0.05 & 0.04 & 5 & 0.86 & 0.027 \\
$\mathrm{~Pb}$ & $<\mathrm{DL}$ & $<\mathrm{DL}$ & $<\mathrm{DL}$ & 5 & 0.37 & 0.30 \\
$\mathrm{Hg}$ & $<\mathrm{DL}$ & $<\mathrm{DL}$ & $<\mathrm{DL}$ & 0.2 & 0.025 & 0.002 \\
\hline
\end{tabular}

\subsubsection{Dimensional Stability}

The length change data show that the grouted waste forms, whether solidifying the liquid waste as an acidic solution, pretreated to a basic solution (alkaline grout), or calcined to a solid (denitrated grout), have varying degrees of stability (Table 14). The denitrated waste form showed the greatest stability with no length change. This is likely the result of the waste solution already solidified (thus more easily solidified and stabilized in grout) and more stable than the liquid waste forms. Shrinkage was observed with the acidic grouted waste form indicating internal stresses that likely caused weakening of the grout matrix when subjected to thermal cycling and water immersion. These stresses are in a normal range and by itself would not cause concern. This stress data coupled with the other tests results explain some of the observed spalling and cracking of the acidic grout. Alkaline grout showed only minor shrinkage. 
Table 14. Percent length change over 28-day period. Acidic grout had the greatest amount of length change show internal stress being formed while curing.

\begin{tabular}{ccc}
\hline Grouted Waste Form Type & $\begin{array}{c}\text { Waste Loading } \\
(\mathrm{wt} \%)\end{array}$ & $\begin{array}{c}\text { Length Change } \\
(\%)\end{array}$ \\
\hline Acidic & 17 & -0.16 \\
Alkaline & 22 & -0.08 \\
Acidic & 23 & -0.18 \\
Denitrated & 30 & 0.00 \\
Acidic & 33 & -0.09 \\
\hline
\end{tabular}

\subsection{Summary}

The highly acidic, alkali nitrate waste solutions at the INEEL can be grouted at a low $\mathrm{pH}$ $(\mathrm{pH}<1.0)$ and exceed all requirements for 28-day compressive strength, the TCLP retention of toxic elements and performs comparably for nitrate leach resistance. The requirements followed in this study are those stated in by the NRC in the Technical Position on Waste Form. These conservative qualifying factors for 28-day compressive strength, thermal cycle integrity, and 90-day immersion endurance guide result in a safe and solid grouted waste form that can be re-deposited most anywhere. The grouted waste forms use commonly available grout forming products: blast furnace slag, portland cement, and class $\mathrm{F}$ fly ash. By using a grout mixture high in slag, heavy metals are rendered less leachable and nitrates are tied up in the tighter microstructure.

Even though the acidic grout waste forms did not pass the 90-day immersion test and do not meet $\mathrm{NRC}$ technical guidelines, the grouted forms could still be disposed in a site with waste acceptance criteria dealing only with leachable toxic elements because they pass TCLP. In addition, it is felt that if the immersion test were extended to 


\section{SOLIDIFICATION OF ACIDIC, HIGH-SODIUM LOW LEVEL WASTE AT THE IDAHO NATIONAL ENGINEERING AND ENVIRONMENTAL LABORATORY'13}

High-activity wastes at the Idaho Nuclear Technology and Engineering Center, INTEC (previously known as the Idaho Chemical Processing Plant, ICPP) include about $6800 \mathrm{~m}^{3}$ of aqueous acidic tank waste and $3800 \mathrm{~m}^{3}$ of solid dry calcine. As part of the Settlement Agreement with the State of Idaho, the Idaho National Engineering and Environmental Laboratory (INEEL) is required to cease use of all existing liquid high-activity waste storage tanks at ICPP by 2015. The High-Level Waste Program Plan calls for the tank waste to be separated via solvent extraction and ion exchange into high-activity and low-activity radioactive wastes. The small volume, high-activity waste will be vitrified and disposed of in a geological repository. The large volume, low-level waste (LLW) will be grouted and sent to a nearsurface low-level disposal facility.

This study has been concerned with the development of a grout formulation and methodology for the solidification of the low-level waste, with particular emphasis on the 'sodium-bearing tank waste'-a highly acidic waste containing a high concentration of sodium and other metal nitrates, including hazardous metals and radionuclides. At this stage, a range of waste pre-treatment/grout options are being considered given that the size / location of the disposal facility and the disposal cost are not known. These options are: 1) grouting thermally calcined LLW (destruction of the nitrates), hereafter called denitrated LLW grout; 2) grouting of waste pre-conditioned to $\mathrm{pH}<2$, hereafter called acidic LLW grout; and 3) grouting of waste pre-conditioned to $\mathrm{pH} \geq 12$, hereafter called alkaline LLW grout.

Through funding from the Office of Science and Technology (EM50), U.S. Department of Energy, AEA Technology is assisting INEEL in these programs by transferring its experience from all stages of waste cementation process and product development.

\subsection{Experimental Methods}

Work to date has focused on the use of inactive, simulated sodium-bearing waste. The general composition is shown in Table 15.

Three grout formulations were tested for solidifying and stabilizing the simulated waste. The formulations are presented in Table 16. Denitrated LLW grout was prepared by pre-treating the simulant via thermal calcination at $650^{\circ} \mathrm{C}$ to destroy nitrates. The solid product is then ground to sand-size and grouted with equal parts of Portland cement (PC), blast furnace slag (BFS), and coal fly ash (Class F). Water is added at a water-to-total solids ratio of 0.40 and the waste loading is 25 weight per cent (wt \%).

Acidic LLW grout is prepared by partially neutralizing the acidity with sodium hydroxide to $\mathrm{pH}$ of about 1 , then grouting the liquid with three parts blast furnace slag, one part Portland cement, and one part fly ash. The liquid-to-solids ratio is 0.40 at a waste loading of $30 \mathrm{wt} \%$.

Alkaline LLW grout is prepared by adding sodium hydroxide and calcium hydroxide to produce solution with a $\mathrm{pH}$ of $\sim 12$. At this $\mathrm{pH}$, the sodium hydroxide removes soluble aluminum from solution and the calcium hydroxide removes from solution components that retard cement setting (for example, fluoride). The liquid is then grouted with nine parts blast furnace slag and one part Portland cement. No water is added; thus, the liquid-to-solids ratio is 0.83 . The waste loading of $42.5 \mathrm{wt} \%$ is limited to a sodium nitrate to total cement mass ratio of 0.2 by weight. 
Table 15. Simulant Concentrations.

\begin{tabular}{|c|c|c|}
\hline Species & $\begin{array}{c}\text { Concentration of species } \\
\text { (molarity) }\end{array}$ & $\begin{array}{l}\text { Simulant } \\
\text { Chemical }\end{array}$ \\
\hline $\mathrm{H}^{+}$ & $7.70 \mathrm{E}-01$ & Combination of acids (see below) \\
\hline $\mathrm{Al}^{3+}$ & 3.37E-01 & $\mathrm{Al}\left(\mathrm{NO}_{3}\right)_{3}$ \\
\hline $\mathrm{As}^{5+}$ & $3.11 \mathrm{E}-05$ & $\mathrm{As}_{2} \mathrm{O}_{5}$ \\
\hline $\mathrm{Ba}^{2+}$ & $3.21 \mathrm{E}-05$ & $\mathrm{Ba}\left(\mathrm{NO}_{3}\right)_{2}$ \\
\hline $\mathrm{B}^{3+}$ & $9.47 \mathrm{E}-03$ & $\mathrm{H}_{3} \mathrm{BO}_{3}$ \\
\hline $\mathrm{Cd}^{2+}$ & $1.47 \mathrm{E}-03$ & $\mathrm{Cd}\left(\mathrm{NO}_{3}\right)_{2} \bullet 4 \mathrm{H}_{2} \mathrm{O}$ \\
\hline $\mathrm{Ca}^{2+}$ & $2.79 \mathrm{E}-02$ & $\mathrm{Ca}\left(\mathrm{NO}_{3}\right)_{2}$ \\
\hline $\mathrm{Cl}^{-}$ & $1.53 \mathrm{E}-02$ & $\mathrm{HCl}$ \\
\hline $\mathrm{Cr}^{3+}$ & $1.95 \mathrm{E}-03$ & $\mathrm{Cr}\left(\mathrm{NO}_{3}\right)_{3} \bullet 9 \mathrm{H}_{2} \mathrm{O}$ \\
\hline $\mathrm{Cs}^{+}$ & $5.79 \mathrm{E}-10$ & $\mathrm{CsNO}_{3}$ \\
\hline $\mathrm{F}^{-}$ & $3.63 \mathrm{E}-02$ & $\mathrm{HF}$ \\
\hline $\mathrm{Fe}^{3+}$ & $1.16 \mathrm{E}-02$ & $\mathrm{Fe}\left(\mathrm{NO}_{3}\right)_{3} \bullet 9 \mathrm{H}_{2} \mathrm{O}$ \\
\hline $\mathrm{Pb}^{2+}$ & 7.37E-04 & $\mathrm{Pb}\left(\mathrm{NO}_{3}\right)_{2}$ \\
\hline $\mathrm{Mn}^{2+}$ & $8.42 \mathrm{E}-03$ & $\mathrm{Mn}\left(\mathrm{NO}_{3}\right)_{2}$ \\
\hline $\mathrm{Hg}^{2+}$ & 5.73E-04 & $\mathrm{Hg}\left(\mathrm{NO}_{3}\right)_{2} \bullet \mathrm{H}_{2} \mathrm{O}$ \\
\hline $\mathrm{Mo}^{6+}$ & $3.68 \mathrm{E}-04$ & $\mathrm{H}_{2} \mathrm{MoO}_{4}$ \\
\hline $\mathrm{Ni}^{2+}$ & $8.42 \mathrm{E}-04$ & $\mathrm{Ni}\left(\mathrm{NO}_{3}\right)_{2} \bullet 6 \mathrm{H}_{2} \mathrm{O}$ \\
\hline $\mathrm{NO}_{3}^{-}$ & $2.67 \mathrm{E}+00$ & $\mathrm{HNO}_{3}$ \\
\hline $\mathrm{PO}_{4}{ }^{3-}$ & 0 & $\mathrm{H}_{3} \mathrm{PO}_{4}$ \\
\hline $\mathrm{K}^{+}$ & $1.08 \mathrm{E}-01$ & $\mathrm{KNO}_{3}$ \\
\hline $\mathrm{Se}^{4+}$ & $1.50 \mathrm{E}-05$ & $\mathrm{SeO}_{2}$ \\
\hline $\mathrm{Ag}^{+}$ & $1.21 \mathrm{E}-05$ & $\mathrm{AgNO}_{3}$ \\
\hline $\mathrm{Na}^{+}$ & $1.10 \mathrm{E}+00$ & $\mathrm{NaNO}_{3}$ \\
\hline $\mathrm{Sr}^{2+}$ & 0 & $\mathrm{Sr}\left(\mathrm{NO}_{3}\right)_{2}$ \\
\hline $\mathrm{SO}_{4}^{2-}$ & $2.63 \mathrm{E}-02$ & $\mathrm{H}_{2} \mathrm{SO}_{4}$ \\
\hline $\mathrm{Zr}^{4+}$ & $5.26 \mathrm{E}-04$ & $\mathrm{ZrO}\left(\mathrm{NO}_{3}\right)_{2} \bullet 3 \mathrm{H}_{2} 0$ \\
\hline
\end{tabular}


Table 16. Estimated grout formulations to produce a cubic meter of grouted low-activity waste.

\begin{tabular}{|c|c|c|c|c|c|c|c|c|c|}
\hline Grout Type & $\begin{array}{c}\text { Liquid } \\
\text { LLW } \\
\left(\mathrm{m}^{3}\right)\end{array}$ & $\begin{array}{c}\text { Mass } \\
\text { LLW }\end{array}$ & $\begin{array}{c}50 \mathrm{w} / \mathrm{o} \\
\mathrm{NaOH} \\
(\mathrm{kg})\end{array}$ & $\begin{array}{c}\mathrm{Ca}(\mathrm{OH})_{2} \\
(\mathrm{~kg})\end{array}$ & $\begin{array}{c}\text { Waste } \\
\text { Loading } \\
(\mathrm{wt} \%)\end{array}$ & $\begin{array}{c}\text { Portland } \\
\text { Cement } \\
(\mathrm{kg})\end{array}$ & $\begin{array}{c}\text { Slag } \\
(\mathrm{kg})\end{array}$ & $\begin{array}{c}\text { Fly } \\
\text { Ash } \\
(\mathrm{kg})\end{array}$ & $\begin{array}{c}\text { Water } \\
(\mathrm{kg})\end{array}$ \\
\hline $\begin{array}{c}\text { Denitrated } \\
\text { LLW Grout }\end{array}$ & 4.00 & $\begin{array}{c}\text { (of denitrated } \\
\text { solids })\end{array}$ & $\mathrm{n} / \mathrm{a}$ & $\mathrm{n} / \mathrm{a}$ & $\begin{array}{c}\text { Solids } \\
25.0\end{array}$ & 284 & 284 & 284 & 510 \\
\hline $\begin{array}{c}\text { Acidic LLW } \\
\text { Grout }\end{array}$ & 0.53 & 587 & 29 & $\mathrm{n} / \mathrm{a}$ & $\begin{array}{c}\text { Liquid } \\
30.0\end{array}$ & 269 & 806 & 269 & 0 \\
\hline $\begin{array}{c}\text { Alkaline } \\
\text { LLW Grout }\end{array}$ & 0.63 & 693 & 101 & 117 & $\begin{array}{c}\text { Liquid } \\
42.5\end{array}$ & 72 & 647 & 0 & 0 \\
\hline
\end{tabular}

(Table based on scale-up, not actual results)

The criteria used to judge the success of the work are the US Nuclear Regulatory Commission's branch technical position on low-level wasteform requirements and the Environmental Protection Agency's Toxicity Characteristic Leaching Procedure (TCLP) limits.

\subsection{Waste Product Performance}

\subsubsection{Small-scale studies at INEEL}

A range of grout formulations has been evaluated in small-scale trials undertaken at INEEL. These trials have considered a variety of parameters such as:

Thermal denitration of the waste

Pre-neutralization using sodium hydroxide

Cement blends (PC, BFS, fly ash

Waste loading

Use of superplasticizer

These initial studies have been reported previously ${ }^{5,6}$ and they have demonstrated the necessity of preconditioning the sodium-bearing, low-activity waste prior to grouting. This can take the form of thermal calcination and denitration, reducing the acidity, or rendering the waste chemically alkaline. On-going studies at INEEL are showing that all three processes can produce acceptable LLW grouts, which meet the NRC and EPA requirements as shown in Table 17.

Table 17. Grout test results.

\begin{tabular}{|l|c|c|c|c|c|c|c|}
\hline \multicolumn{1}{|c|}{ Grout Type } & $\begin{array}{c}\text { Waste } \\
\text { Loading } \\
(\text { wt } \%)\end{array}$ & $\begin{array}{c}\text { Cured } \\
\text { Density } \\
\left(\mathrm{g} \mathrm{cm}^{-3}\right)\end{array}$ & $\begin{array}{c}\mathbf{2 8} \text { Day } \\
\text { Strength } \\
(\mathrm{kPa})\end{array}$ & $\begin{array}{c}\text { Thermal } \\
\text { Cycle } \\
\text { Strength } \\
(\mathrm{kPa})\end{array}$ & $\begin{array}{c}90 \text { Day } \\
\text { Immerse } \\
\text { Strength } \\
(\mathrm{kPa})\end{array}$ & $\begin{array}{c}\text { Vol grout } \\
\text { to Vol } \\
\text { waste }\end{array}$ & $\begin{array}{c}\text { Passed } \\
\text { TCLP }\end{array}$ \\
\hline $\begin{array}{l}\text { Denitrated LLW } \\
\text { Grout }\end{array}$ & $\begin{array}{c}\text { Solids } \\
25.0\end{array}$ & 1.82 & $\begin{array}{c}16203 \\
(2350 \mathrm{psi})\end{array}$ & $\begin{array}{c}24614 \\
(3570 \mathrm{psi})\end{array}$ & $\begin{array}{c}16272 \\
(2360 \mathrm{psi})\end{array}$ & 0.26 & Yes \\
\hline Acidic LLW Grout & $\begin{array}{c}\text { Liquid } \\
30.0\end{array}$ & 1.96 & $\begin{array}{c}49642 \\
(7200 \mathrm{psi})\end{array}$ & $\begin{array}{c}36060 \\
(5230 \mathrm{psi})\end{array}$ & $\begin{array}{c}10549 \\
(1530 \mathrm{psi})\end{array}$ & 1.87 & Yes \\
\hline Alkaline LLW Grout & $\begin{array}{c}\text { Liquid } \\
42.5\end{array}$ & 1.63 & $\begin{array}{c}6205 \\
(900 \mathrm{psi})\end{array}$ & $\begin{array}{c}5585 \\
(810 \mathrm{psi})\end{array}$ & $\begin{array}{c}4964 \\
(720 \mathrm{psi})\end{array}$ & 1.44 & Yes \\
\hline
\end{tabular}


Grouting the denitrated solid waste produces a viable grout product at $25 \mathrm{wt} \%$ waste loading; loadings of up to $35 \mathrm{wt} \%$ can be tolerated to meet minimum NRC and EPA standards. The grout exceeds the compressive strength minimum of $3448 \mathrm{kPa}(500 \mathrm{psi})$. The calcination/denitration process was found to destroy about $80 \%$ of the nitrates and reduces the volume 3.8 fold.

It was determined that the sodium-bearing LLW could be grouted by controlling the $\mathrm{pH}$ of the waste solution and increasing the slag content. The waste can be grouted at $0.6<\mathrm{pH}<2$ and at $\mathrm{pH}=12$. For the acid LLW grout, this is done by adding sodium hydroxide to partially neutralize the acidity and then adding slag to the solution prior to the cement powder. It was found that this order of mixing was critical in forming a much stronger grout. This process produces high strength grouts with waste loadings that increase the waste volume to about 1.5 to 2 times the original volume. The short-term results look promising, but the long-term tests have not been completed. The initial 90 day immersion strength test results are above the minimum $3448 \mathrm{kPa}(500 \mathrm{psi})$ at $10549 \mathrm{kPa}$; however, the samples do not maintain $75 \%$ of their initial strength. Thus, extended immersion tests are needed for 180 days to ensure the grout strength does not fall below $3448 \mathrm{kPa}$ as required by the NRC. Acidic grout specimens successfully passed the thermal cycling tests.

The alkaline LLW grout formulation gives a liquid waste loading of $42.5 \mathrm{wt} \%$ and results in a volume increase of about 1.5 times the original volume. The samples passed the thermal cycle and 90 day immersion tests. This formulation has been more extensively studied by AEA Technology, including the production of a large-scale (200 liter) specimen (see later).

All three grouts pass the Toxicity Characteristic Leach Procedure (TCLP) at the more stringent Universal Treatment Standard levels. The samples were tested for nine toxic metals: silver, arsenic, barium, cadmium, chromium, mercury, nickel, lead, and selenium. In general, all metals leached below detection limits, except for barium and chromium. Barium comes from the blast furnace slag and leaches at approximately 10 times less than UTS limits for all three types of grout. Chromium leaches from only the denitrated LLW grout where the calcination/denitration process oxidizes the chromium to a +6 valance. The sulfides in the slag help to reduce chromium to the +3 valance state, which is less leachable. The chromium concentration in the TCLP leachate ranged up to $0.50 \mathrm{mg}^{-1}$; the UTS limit for chromium is $0.86 \mathrm{mg} \mathrm{l}^{-1}$.

\subsubsection{Large-scale studies at AEA Technology (Winfrith, UK)}

As a result of the successful small-scale mixes it was decided that the alkaline LLW grout formulation should be tested at full-scale (200 liters), using AEA Technology's existing grout mixing/testing facilities at Winfrith Technology Center.

The 200-litre specimen was prepared using an in-drum mixing method where dry cement powders (slag + Portland cement + lime) were added to the pre-conditioned (with sodium hydroxide) and lime pre-treated waste slurry. During the mixing process the rotational paddle torque was monitored using an optical torque transducer coupled to a data logger. Once mixing was complete cube and prism samples of the cemented product were cast and stored in controlled temperature and humidity conditions. These test samples allowed long-term testing, by non-destructive and destructive means, of the physical properties of the grout formulation. A frame containing vibrating wire strain gauges and type $\mathrm{K}$ thermocouples was inserted into the cemented product immediately after mixing was completed. The 200-litre steel drum containing the cemented product was sealed and allowed to cure in a controlled environment, nominally at $20 \pm 2^{\circ} \mathrm{C}$ and $80-90 \%$ relative humidity. The cement curing exotherm was monitored over a period of 140 hours and the dimensional movement monitored for 90 days. After 99 days curing the 200-litre drum was cored and sectioned for testing and examination. 
The large-scale mix was very successful. During mixing the torque increased as expected with powder addition, peaking at $18.4 \mathrm{Nm}$ immediately after powder addition finished and falling to $13 \mathrm{Nm}$ after a further 20 minutes mixing. The viscosity of the grout/waste mix was $0.66 \mathrm{~Pa} \mathrm{~s}(660 \mathrm{cp})$.

The setting time of the cemented product was in the range: less than 35 hours but greater than 24 hours. Zero bleed was recorded on the sample after 24 hours curing. The results from the implanted thermocouples show that the maximum temperature in the drum center was $42^{\circ} \mathrm{C}$ after 35 hours curing. This compares favorably with other similar cemented radioactive waste sludges. The density of the cemented product after two days curing was $1.65 \mathrm{~g} \mathrm{~cm}^{-3}$.

The results from the physical testing of the cured specimens taken from the wet mix and the drum sample itself are given in Table 18. The ultrasonic pulse velocity of cube samples cast from the 200-litre mix shows a steady increase from $1.64 \mathrm{~km} \mathrm{~s}^{-1}$ at two days to $2.66 \mathrm{~km} \mathrm{~s}^{-1}$ at 90 days. Such behavior is indicative of a progressive strength development. It is therefore not surprising that the compressive strength of the cube samples increased from $0.5 \mathrm{MPa}$ (73 psi) at two days to $9.5 \mathrm{MPa}(1378 \mathrm{psi})$ at 90 days. Another indicator of the strength development of the mix is the elastic modulus of prism samples which also show a progressive increase from $0.99 \mathrm{GPa}$ at two days to $7.75 \mathrm{GPa}$ at 90 days.

The dimensional stability of prism samples cast from the 200-litre mix was in the range 53 microstrain shrinkage at seven days to 509 microstrain shrinkage at 90 days. (Dimensional stability is quoted in units of microstrain which represent $10^{-4}$ per cent movement or one part per million. Thus a shrinkage movement of 500 microstrain is equivalent to $0.5 \mathrm{~mm}$ contraction over one meter of grout.) Between 42 and 90 days curing little significant movement was detected. The dimensional stability of the 200 -litre drum sample was monitored using the implanted vibrating wire gauges. Both horizontal and vertical gauges show an initial expansion probably due to thermal expansion. Thereafter both gauges showed progressive shrinkages of 295 and 360 microstrain respectively over the 90 day curing period.

Mercury intrusion porosimetry was used to analyze samples of the cemented product for pore size distribution at 43 days and 113 days curing. In addition a sample of the full-scale cemented product was analyzed at 113 days curing to allow comparison to the small-scale samples. The results are shown in Table 19. The porosity of all three samples is approximately the same, in the region of $20-30 \%$.

However, the median pore diameter and total pore area data show a shift to smaller pore sizes as the age of the samples increase - this is consistent with the gradual filling of pores with hydration products as the age of the sample increases and hydration continues. Comparison of the small and full-scale samples tested at 113 days suggests a slightly more advanced state of cement hydration for the full-scale sample, probably due to the initial acceleration of hydration of this sample due to the higher curing exotherm compared with the small-scale specimens.

After 99 days curing the drum sample was cored and subsequently a $110^{\circ}$ section of the drum sample was cut out. The overall quality of the final product can be seen from the photograph of the sectioned drum sample (see Figure 2). 
Table 18. Test results for the full-scale (200-litre) cementation trial on alkaline sodium bearing waste simulant grout formulation.

\begin{tabular}{|c|c|c|c|c|c|c|}
\hline $\begin{array}{l}\text { Curing } \\
\text { Time } \\
\text { (Days) } \\
\end{array}$ & $\begin{array}{l}\text { Compressive } \\
\text { Strength } \\
(\mathrm{Mpa}) \\
\end{array}$ & $\begin{array}{l}\text { Ultrasonic Pulse } \\
\text { Velocity } \\
\left(\mathrm{km} \mathrm{s}^{-1}\right)\end{array}$ & $\begin{array}{c}\text { Elastic } \\
\text { Modulus } \\
(\mathrm{GPa})\end{array}$ & $\begin{array}{c}\text { * Dimensional } \\
\text { Stability } \\
\text { (Microstrain) }\end{array}$ & $\begin{array}{l}\text { * Dimensional } \\
\text { Stability of } \\
2001 \text { Sample - } \\
\text { Horizontal } \\
\text { Gauge } \\
\text { (Microstrain) }\end{array}$ & $\begin{array}{c}\text { * Dimensional } \\
\text { Stability of } \\
2001 \text { Sample - } \\
\text { Vertical } \\
\text { Gauge } \\
\text { (Microstrain) }\end{array}$ \\
\hline 1 & - & - & - & - & +316 & +365 \\
\hline 2 & 0.5 & 1.64 & 0.99 & 0 & +224 & +273 \\
\hline 7 & 3.7 & 2.34 & 5.22 & -53 & +220 & +243 \\
\hline 14 & - & 2.45 & 6.22 & -147 & +172 & +186 \\
\hline 21 & - & 2.49 & 6.67 & -112 & +133 & +155 \\
\hline 28 & 6.7 & 2.54 & 6.91 & -258 & +108 & +127 \\
\hline 42 & - & 2.58 & 7.23 & -494 & +90 & +95 \\
\hline 56 & - & 2.60 & 7.46 & -498 & +60 & +55 \\
\hline 90 & 9.5 & 2.66 & 7.75 & -509 & +21 & +5 \\
\hline 1 to 90 & - & - & - & -509 & -295 & -360 \\
\hline
\end{tabular}

Table 19. Pore size distribution results for full-scale (200-litre) cementation trial on alkaline sodium bearing waste simulant grout formulation.

\begin{tabular}{|c|c|c|c|c|}
\hline Age (Days) & $\begin{array}{c}\text { Total Porosity } \\
(\%)\end{array}$ & $\begin{array}{c}\text { Threshold Pore } \\
\text { Diameter } \\
(\mu \mathrm{m})\end{array}$ & $\begin{array}{c}\text { Median Pore } \\
\text { Diameter } \\
(\mu \mathrm{m})\end{array}$ & $\begin{array}{c}\text { Total Pore Area } \\
\left(\mathrm{m}^{2} \mathrm{~g}^{-1}\right)\end{array}$ \\
\hline 43 & 20.6 & 0.43 & 0.28 & 2.68 \\
\hline 113 & 22.0 & 0.46 & 0.24 & 4.34 \\
\hline $\begin{array}{c}113 \\
\text { (Full-scale sample) }\end{array}$ & 22.5 & 0.37 & 0.21 & 4.67 \\
\hline
\end{tabular}

Visual inspection showed the product to be homogenous with no unmixed areas of waste or cement powder. The sample also contained an even distribution of lime particles from the lime pre-treatment stage. Voidage measurement by bubble count was very low with an estimated macrovoidage of less than $0.5 \%$. Some hairline cracks can be seen around the exposed strain gauges in the drum center and some slight hairline cracking at the edge of the drum at the position of the rolling rings. This hairline cracking is not uncommon and is usually an artifact of the presence of the measuring gauge and/or handling of the drum. It does not indicate a significant deterioration in the integrity of the drum product. The surfaces of the sectioned sample were free from major cracking which would threaten the integrity and monolithicity of the sample. Some damage sustained during the final stages of sectioning is visible in the base of the sample. 




Figure 2. Cored full-scale (200 L) cemented sodium-bearing waste. 
The non-destructive and destructive test results for the three full depth cores are given in Table 20 . Each core is cut into at least three pieces to allow assessment of the grout performance in the top, middle and bottom sections of the drum product. The results show relatively consistent values of density and ultrasonic pulse velocity, with average values of $1.63 \mathrm{~g} \mathrm{~cm}^{-3}$ and $2.63 \mathrm{~km} \mathrm{~s}^{-1}$. In-situ cube strengths, calculated from the measured compressive strengths of the core samples show some variation with an average value of $7.9 \mathrm{MPa}(1146 \mathrm{psi}$ ) and a standard deviation of $1.2 \mathrm{MPa}$. The value of $5.6 \mathrm{MPa}$ measured for the base sample from core number 2 is significantly less than the other eight measured values but is not considered representative. It is likely that the coring process resulted in some physical damage to this specific sample.

Table 20. Testing cores from full-scale (200-litre) cementation trial on alkaline sodium bearing waste simulant grout formulation.

\begin{tabular}{|c|c|c|c|c|}
\hline Core Number & Position in drum & $\begin{array}{c}\text { In-situ Cube Strength } \\
(\mathrm{MPa})\end{array}$ & $\begin{array}{c}\text { Density } \\
\left(\mathrm{g} \mathrm{cm}^{-3}\right)\end{array}$ & $\begin{array}{c}\text { Pulse Velocity } \\
\left(\mathrm{km} \mathrm{s}^{-1}\right)\end{array}$ \\
\hline & Top & 8.6 & 1.62 & 2.62 \\
\hline 1 & Middle & 7.6 & 1.63 & 2.62 \\
\hline & Base & 7.5 & 1.64 & 2.62 \\
\hline 2 & Top & 8.9 & 1.62 & 2.63 \\
\hline & Middle & 7.6 & 1.63 & 2.62 \\
\hline & Base & 5.6 & 1.64 & 2.63 \\
\hline 3 & Top & 8.5 & 1.63 & 2.65 \\
\hline & Middle & 7.4 & 1.63 & 2.65 \\
\hline & Base & 9.8 & 1.63 & 2.65 \\
\hline
\end{tabular}

All samples cut to $200 \mathrm{~mm}$ length $\mathrm{x} 92 \mathrm{~mm}$ diameter

\subsection{Implications for Waste Processing}

Many of the mixes produced during the earlier small-scale studies were scoping trials where the materials were mixed by hand and data was not collected on the viscosity of the mix. However, as part of AEA Technology's scaling-up of the alkaline LLW grout formulation the viscosity of the grout mix was recorded and it indicated that the mix could be effectively mixed at large-scale; an observation confirmed by the successful production of the 200 liter drum specimen.

In-drum mixing is one option for the eventual waste processing strategy at INEEL, but the 1.5 times volume increase associated with the alkaline LLW grout formulation may lead to an unacceptably high number of waste product drums. An alternative option is to pump the fluid grouted waste mixture into one of the emptied silos that had previously contained the sodium bearing waste. The objective being to produce a massive cemented wasteform which is capable of meeting the appropriate regulations (see earlier) in its own right, but would benefit from the additional containment afforded by the silo. This option would also avoid the need to decommission the existing silos after they were emptied. However, for this option to work, the grouted waste must have sufficient fluidity to permit pumping over large distances whilst still retaining the ability to eventually set and form a good quality wasteform. Results to date indicate that this option may be a possibility, but considerably more work would be have to be undertaken to prove that it is a viable alternative to the production of drummed wasteforms. 


\subsection{Conclusions and Future Work}

Small-scale trial mixes have demonstrated three grouting options, namely the denitrated LLW grout, the acidic LLW grout and the alkaline LLW grout. All produce samples that satisfy regulatory requirements (with the potential exception of the 90 day immersion test for the LLW acidic grout formulation). In addition, the alkaline LLW grout formulation has been used to produce a 200 liter product with a $42.5 \mathrm{wt} \%$ waste loading that fully meets or exceeds regulatory requirements.

Current cost projections utilize the cost of disposal and storage as the dominant cost factor. Thus disposal volume is a critical driver. If the grout goes to drums for later disposal, preliminary cost estimates show the denitration process to be the most cost effective due to the greatest volume reduction. This includes adjustments for the higher process energy costs and off-gas treatment. However, if volume ceases to be the main driver, the alternative processes of liquid waste grouting would be more cost effective.

As part of the decision making process, future work programs will seek to address:

- Maximizing the waste loading for each of the waste pre-treatment/grout options

- $\quad$ Optimization of the grout process, e.g., process control parameters and operating limits

- $\quad$ Production of full-scale data to meet NRC requirements - this will build upon full-scale (200 liter drum) data already collated for the alkaline grout option

- $\quad$ Consideration of associated waste streams in the ICPP, e.g., aluminum and zirconium calcine streams. 


\section{REFERENCES}

1. Lockheed Martin Idaho Technologies Co., "High-Level Waste Program Plan," INEL-96/122, April 1996.

2. U. S. Nuclear Regulatory Commission Technical Branch of the Low-Level Waste Management and Decommissioning Division, "Technical Position on Waste Form," Revision 1, January, 1991.

3. Lockheed Martin Idaho Technologies Co., High-Level Waste Program Plan, ONEL-96/122, April 1996.

4. D. Singh, et al., "Low-Temperature-Setting Phosphate Ceramics for Stabilizing DOE Problem Low-Level Mixed Waste," WM '94, Proc. Symposium on Waste Management, Tucson AZ (1994) p. 1853.

5. Herbst, A. K., "Optimization of Hydraulic Cement Ad Mixture Waste Forms for Sodium-Bearing, High Aluminum, and High Zirconium Wastes," $18^{\text {th }}$ Annual U. S. Department of Energy Low-Level Radioactive Waste Management Conference Proceedings, May 20-22, 1997.

6. Herbst, A. K., D. W. Marshall, and J. A. McCray, "Idaho Chemical Processing Plant Low-Activity Waste Grout Stabilization Development Program FY-97 Status Report," INEEL/EXT-98-00116, February 1998.

7. B. C. Spaulding, et al., "INTEC TFF Tanks WM-182 and WM-183 Closure Study," INEEL/EXT-98-00913, September 1998.

8. Sawyer, J. L., "Volume Change, Chapter 16," Significance of Tests and Properties of Concrete and Concrete-Making Materials, Philadelphia, PA, American Society for Testing and Materials.

9. Neville, A. M., Properties of Concrete, New York: John Wiley and Sons, Inc., 1997.

10. Palmer, J. D., and D. L. Smith, Incorporation of Low and Medium Level Radioactive Wastes (Solids and Liquids) in Cement, Final Report, UKAEA/EUR10561EN, 1986.

11. ASTM C 490-96, "Standard Practice for Use of Apparatus for the Determination of Length Change of Hardened Cement Paste, Mortar, and Concrete," American Society for Testing and Materials.

12. Mattus, C. H., and T. M. Gilliam, A Literature Review of Mixed Waste Components: Sensitivities and Effects Upon Solidfication/Stabilization in Cement-Based Matrices, ORNL/TM-12656.

13. Simmons, R. F., S. J. Palethorpe, A. K. Herbst, and D. W. Marshall, "Solidification of Acid, High-Sodium Low Level Waste at the Idaho National Engineering and Environmental Laboratory," Proceedings of Spectrum '98 International Conference on Decommissioning and Decontamination and on Nuclear and Hazardous Waste Management, Denver, Colorado, pages 689-696, September 13-18, 1998. 